\title{
Enhancement of a photovoltaic cell performance by a coupled cooled nanocomposite thermoelectric hybrid system, using extended thermodynamics
}

\author{
H. Machrafi \\ Université Libre de Bruxelles, Service Chimie-Physique, Ecole Polytechnique, CP165/62, \\ avenue F.D. Roosevelt, 50, 1050 Brussels, Belgium \\ E-mail : $\underline{\text { Hatim.Machrafi@ulb.ac.be }}$
}

\begin{abstract}
A new analytical mathematical model is developed, describing a cooled photovoltaicthermoelectric hybrid system. The thermoelectric material is a nanocomposite where the model takes into account size-dependent non-local thermoelectric properties from an extended thermodynamic point of view. The photovoltaic device powers also the cooling system. The model determines first the optimum thickness of the photovoltaic device, then studies the influence of several size-related parameters on the thermoelectric efficiency (also related to the figure of merit) and finally, coupled to a cooling device, the overall efficiency. For the photovoltaic part, the model is applied to two materials, mono-crystalline and poly-crystalline silicon. The thermoelectric part of the model is applied to an n-leg nanocomposite made out of $\mathrm{Sb}_{2} \mathrm{Te}_{3}$ nanoparticles in a $\mathrm{Bi}_{2} \mathrm{Te}_{3}$ matrix and of a p-leg nanocomposite made out of $\mathrm{Bi}_{2} \mathrm{Se}_{3}$ nanoparticles in $\mathrm{a} \mathrm{Bi}_{2} \mathrm{Te}_{3}$ matrix. An optimal total photovoltaic device size has been found to be around $127 \mu \mathrm{m}$ and $1.25 \mu \mathrm{m}$ for the mono- and poly-crystalline silicon, respectively, leading to efficiencies up to $20 \%$, depending on photovoltaic recombination characteristics. With the cooling device, the overall efficiency was increased by up to an additional $10 \%$ (an increase of almost $50 \%$ ), leading to overall efficiencies around $25 \%$.
\end{abstract}

Key words: Photovoltaic power output, Thermoelectric element, Cooled hybrid system, Nanocomposite, Extended irreversible thermodynamics

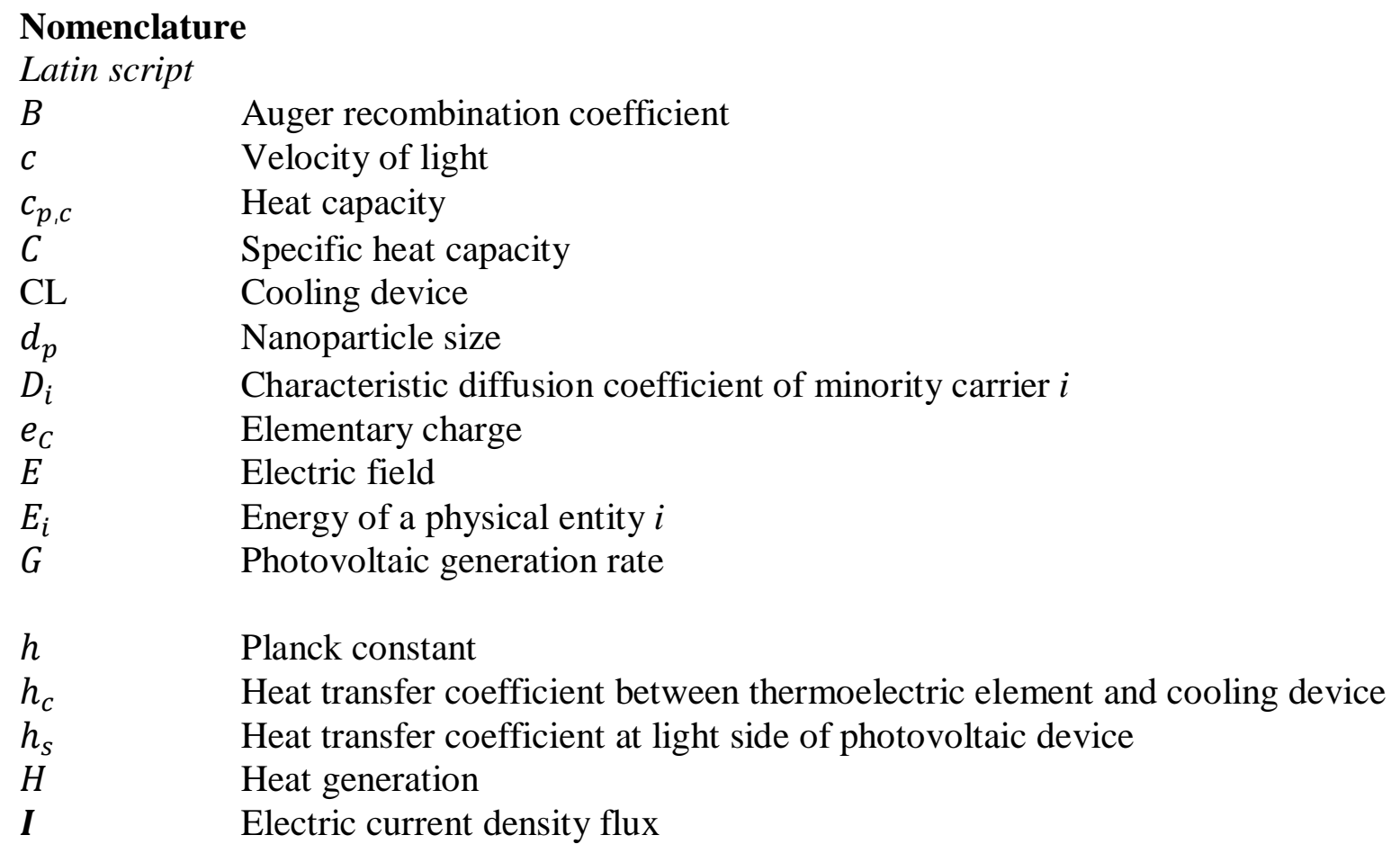




\begin{tabular}{|c|c|}
\hline$I_{A M 1.5}$ & Incident photon energy per surface per wavelength \\
\hline$J^{S}$ & Entropy flux \\
\hline$k$ & Wavenumber \\
\hline$k_{B}$ & Boltzmann constant \\
\hline$K n$ & Knudsen number \\
\hline$l_{i}$ & Correlation length of order $i$ \\
\hline$L$ & Thermoelectric element length \\
\hline$L_{c}$ & Length of contact between thermoelectric element and cooling device \\
\hline$L_{e}$ & Lorentz constant \\
\hline$L_{i}$ & Characteristic diffusion length of minority carrier $i$ \\
\hline$n$ & Electron carrier concentration \\
\hline$n_{i e}$ & Equilibrium carrier concentration \\
\hline$N_{A}$ & Acceptor dopant concentration \\
\hline$N_{C}$ & Density of states for the conduction band \\
\hline$N_{D}$ & Donor dopant concentration \\
\hline$N_{V}$ & Density of states for the valence band \\
\hline$p$ & Hole carrier concentration \\
\hline$P_{c}$ & Power needed for cooling device \\
\hline$P_{e}$ & Electric power \\
\hline PV & Photovoltaic device \\
\hline PVTE & Photovoltaic-thermoelectric hybrid device \\
\hline PVTECL & Photovoltaic-thermoelectric-cooling hybrid device \\
\hline $\operatorname{Pr}$ & Prandtl number \\
\hline$q$ & Heat flux \\
\hline$Q$ & Heat supply to thermoelectric element \\
\hline $\boldsymbol{Q}^{(i)}$ & Heat flux of order $i$ \\
\hline $\boldsymbol{r}$ & Spatial variable in Fourier transformation \\
\hline$r_{p}$ & Nanoparticle radius \\
\hline$R$ & Thermal boundary resistance coefficient \\
\hline$R_{i}$ & Recombination rate with $i=p, n$ \\
\hline$R e$ & Reynolds number \\
\hline$s$ & Entropy function \\
\hline$S$ & Seebeck coefficient \\
\hline$S_{A M 1.5}$ & Standard number of incident photons per surface per wavelength \\
\hline$S_{r}$ & Surface recombination velocity \\
\hline$t$ & Time \\
\hline$T$ & Temperature \\
\hline TE & Thermoelectric element \\
\hline$u$ & Internal energy \\
\hline$v$ & Velocity \\
\hline$v_{c}$ & Velocity of cooling fluid \\
\hline$V$ & Electric potential \\
\hline $\boldsymbol{V}()$ & Space of state variables between the parentheses \\
\hline$V_{D}$ & Built-in voltage \\
\hline$W_{c}$ & Width of cooling device \\
\hline$W_{i}$ & Thickness of $\mathrm{p}(i=p)$ and $\mathrm{n}$-sides $(i=n)$ of the photovoltaic device \\
\hline$y$ & Space coordinate \\
\hline$z$ & Space coordinate \\
\hline$Z T$ & Figure of merit \\
\hline
\end{tabular}


$\alpha$

$\alpha_{i}$

$\gamma_{i}$

$\Gamma_{i}$

$\delta$

$\varepsilon_{0}$

$\varepsilon_{r}$

$\eta_{i}$

$\lambda$

$\Lambda$

$\mu_{c}$

$\mu_{i}$

$\Pi$

$\rho$

$\varrho$

$\sigma^{s}$

$\tau$

$\varphi$

$\chi_{i}$

\section{Subscripts}

0

h

$h p$

A

Aug

I

$L$

SRH

$T$

$a m b$

$b$

$c$

coll

$d$

dop

$e$

env

$g$

j

lp

$\max$

$\min$

$n$

opt

$p$

$p h$
Absorption coefficient of the photovoltaic material

Thermal boundary resistance number $(i=p h, e)$

Phenomenological constant related to relaxation times of order $i$

Phenomenological constant related to correlation lengths of order $i$

Photovoltaic device thickness

Vaccuum permittivity

Relative permittivity

Efficiency $(i=p v, t e, p v t e, c, t o t)$

Thermal conductivity

Mean free path

Dynamic viscosity of the cooling fluid

Mobility of minority carrier $i$

Peltier coefficient

Density

Density of electric charge

Entropy production

Relaxation time

Volume fraction

Phenomenological constant related to transport coefficient of order $i$

Reference or equilibrium

Hot side of the thermoelectric element

Photons with energy higher than bandgap one

Auger

Auger

Current density

Light-generated

Shockley-Read-Hall

Total

Ambient

Bulk

Cooling device or cold side of the thermoelectric element

Collision

Depletion region

Dopant

Electron

Environment (might be equal to ambient, but not necessarily)

Band gap

Joule heating

Photons with energy lower than bandgap one

Maximum

Minimum

Electron carrier

Optimum

Hole carrier

Phonon 


$\begin{array}{ll}\text { photon } & \text { Photon energy } \\ \text { pv } & \text { Photovoltaic device } \\ \text { pvte } & \text { Photovoltaic-thermoelectric hybrid } \\ r e f & \text { Reference } \\ s & \text { Solar } \\ s c & \text { Short-circuit } \\ s f & \text { Surface recombination } \\ t & \text { Time derivative } \\ t e & \text { Thermoelectric element } \\ \text { tot } & \text { Total } \\ & \\ \text { Superscripts } & \\ m & \text { Matrix bulk phase } \\ p & \text { Nanoparticle phase } \\ & \\ \text { Accents on top of character } \\ \cdot & \text { Time derivative } \\ - & \text { Renomarlization } \\ \wedge & \text { Fourier transform }\end{array}$

\section{Introduction}

Developments in renewable energy seek to alleviate the global energy crisis and reduce its impact on the environment. One way is to use solar energy. By means of photovoltaic (PV) solar cells, photonic energy is mainly converted into electricity and waste heat. PV cells have relatively low conversion efficiency, because they can only utilize part of the incident solar energy due to its given bandgap, and require often hybrid configurations [1]. One way to increase the efficiency of PV cells is using thermal management by means of heat sinks [2]. Otherwise, the waste heat can be used in order to be converted to more electricity via thermoelectric devices (TE) [3-6]. As a common PV cell converts a large amount of solar irradiant energy into heat, a hybrid PV cell and TE device (PVTE) may be a prospective way to improve the overall efficiency of solar energy [7]. One form of PVTE systems uses the socalled spectrum splitting concentrating system, where the photons with an energy out of the PV working waveband are incident to the TE devices, generating thereby electricity via the thermoelectric effect [8-10]. This system is complex and the heat produced from the PV is still not used. Connecting the TE device directly at the dark side of the PV cell is simpler and theoretically all thermal energy can be used by the TE device [11], of which the efficiency can be even increased by cooling the TE device [7, 12]. It is the latter hybrid system that we consider in this work. As the efficiency in TE devices are proportional (though not necessarily linearly) on mainly the temperature difference across the device and the figure of merit, both are to be increased. The temperature difference stems from the operating system, while the figure of merit depends on material properties. For this purpose, we propose to use a nanocomposite TE device, so that the figure of merit can be considerably increased, even doubled $[5,6]$. The thermoelectric transport properties are derived by means of a new extended irreversible thermodynamic (EIT) model [5, 13, 14].

The optimization of the overall efficiency of cooled PVTE systems is quite complicated and many works have investigated this mostly by experiments [15-18] or by numerical methods [7, $8,9]$, to mention a few. Hereby, it appeared that working at nanoscale lengths the efficiency could be increased [16]. Analytical models have also been developed, focusing sometimes on optical and electrical modeling [17], but at the cost of simplified physical phenomena [15, 17, 
18]. We intend to develop an analytical model that is easy to use, but still capture complex coupled optic, thermal and electric phenomena that are present in cooled PVTE systems with nanocomposite materials. Such a model can also be used as support for understanding the mechanisms responsible for the performance of photovoltaic and thermoelectric devices.

As for the PV cell, the model takes into account the thickness-dependence of the generation rate, the surface recombination velocity of electron and hole carriers (and indirectly passivation of dangling bonds at the cell's surface), and several recombination mechanisms (SRH, Auger and surface). As for the TE device, the model takes into account, the nanoparticle size, both phonon and electron scattering in both the nanoparticles and the bulk matrix, the nanoparticle volume fraction. In order to maximize the thermoelectric efficiency, not only should the figure of merit be increased by the introduction of nanoparticles [6], but the temperature difference across it should also be optimized by means of a cooling (CL) device right under the TE device. By means of overall thermal modelling, it is the purpose to find optimal conditions for the PVTECL hybrid system. The photovoltaic power, thermoelectric efficiency and overall efficiency are studied as function of several parameters discussed in this paper. Fig. 1 shows a schematic representation of one cell unit of the hybrid system. The photovoltaic device is at the illuminated part on top of the system. Right under it, there is the thermoelectric device, consisting of an n-and p-leg. The hybrid system is cooled by means of a sun-energy powered heat sink.

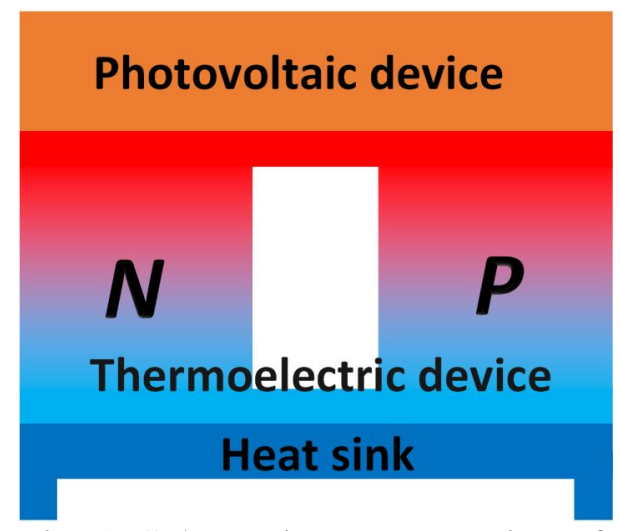

Fig. 1: Schematic representation of a cell unit of the cooled photovoltaic-thermoelectric hybrid system.

The paper is set as follows. Section 2 treats the thermoelectric model, detailing on nanocomposite thermoelectric transport properties. Section 3 treats the EIT model used for nanoscale transport. Section 4 presents the photovoltaic model dealing with opto-electric phenomena, followed by a heat management analysis in Section 5. Section 6 presents a discussion of the material properties and operating conditions. The results are presented in Section 7, concluding in Section 8.

\section{Nanostructured thermoelectric model}

\subsection{Thermoelectric efficiency}

Nanostructured materials can be used in different forms. We are interested here in nanocomposites. Nanocomposites are generally made out of a homogeneous matrix in which nanoparticles are dispersed. For nanoparticles with characteristic lengths of the same order of magnitude or smaller than the phonon and electron mean free paths, the Fourier theory, based on the classical approach of thermodynamics, is not able to predict the thermal interactions in the nanoparticles as well as between the nanoparticle and the bulk material. Therefore, we propose to investigate the problem of heat conduction in nanostructured thermoelectric materials by a more sophisticated thermodynamic formalism, namely Extended Irreversible 
Thermodynamics (EIT) $[5,13,14]$. In this approach, the heat flux is elevated to the status of independent variable at the same footing as the temperature. The total heat flux $\boldsymbol{q}$ is assumed to be the sum of a phonon contribution $\boldsymbol{q}_{\boldsymbol{p}}$ and an electric one $\boldsymbol{q}_{\boldsymbol{e}}$ [6], which are due to the phonon and electron motion through the material:

$q=q_{e}+q_{p h}$

Note that by convention the bold font indicates a vector or higher order tensors of the concerned variable, while scalars are written in normal font. In the presence of an electric field and without local heat supply, the partial energy balances for the phonons and the electrons are

$\frac{\partial u_{p h}}{\partial t}+\nabla \cdot \boldsymbol{q}_{p \boldsymbol{h}}=0$

$\frac{\partial u_{e}}{\partial t}+\nabla \cdot \boldsymbol{q}_{\boldsymbol{e}}=\boldsymbol{I} \cdot \boldsymbol{E}$

with $u_{p h}, u_{e}, \boldsymbol{E}$ and $\boldsymbol{I}$ are the phonon internal energy, electron internal energy, the electric field and the electric current density, respectively. The total energy balance is then, in the absence of a magnetic field, given by

$\frac{\partial u}{\partial t}+\nabla \cdot \boldsymbol{q}=\boldsymbol{I} \cdot \boldsymbol{E}$

with

$u=u_{p h}+u_{e}$

which also implies $C=C_{p h}+C_{e}$, used later on, $C$ being the specific (at constant volume) heat capacity. The continuity law for electric charge is

$\frac{\partial \varrho_{e}}{\partial t}+\nabla \cdot \boldsymbol{I}=0$

with $\varrho_{e}$ the density of electric charge. These equations give us now the evolution of $u_{p h}, u_{e}$ and $\varrho_{e}$, with the corresponding fluxes $\boldsymbol{q}_{\boldsymbol{p} \boldsymbol{h}}, \boldsymbol{q}_{\boldsymbol{e}}$ and $\boldsymbol{I}$. The basic principles of EIT allow postulating additional evolution equations for these fluxes (who are now considered as independent variables) as [6]

$\tau_{p h} \frac{\partial \boldsymbol{q}_{p h}}{\partial t}+\boldsymbol{q}_{p \boldsymbol{h}}=-\lambda_{p h} \nabla T+\Lambda_{p h}^{2} \nabla^{2} \boldsymbol{q}_{p h}$

$\tau_{e} \frac{\partial \boldsymbol{q}_{e}}{\partial t}+\boldsymbol{q}_{\boldsymbol{e}}=-\left(\lambda_{e}+\mathrm{S} \Pi \sigma_{e}\right) \nabla T+\Lambda_{e}^{2} \nabla^{2} \boldsymbol{q}_{\boldsymbol{e}}+\Pi \sigma_{e} \boldsymbol{E}$,

$\tau_{I} \frac{\partial \boldsymbol{I}}{\partial t}+\boldsymbol{I}=\sigma_{e}(\boldsymbol{E}-\mathrm{S} \nabla T)+\Lambda_{I}^{2} \nabla^{2} \boldsymbol{I}$,

where $\tau, \Lambda, \Pi, \mathrm{S}, \lambda$ and $\sigma$ are the relaxation time, mean free path, the Peltier coefficient, the Seebeck coefficient, the thermal conductivity and the electric conductivity, respectively, $T$ the temperature, and the subscripts $p h$ and $e$ standing for phonon and electron, respectively. It should be noted that in Eq. (7a), the phonon transport is assumed to be dominated by normal phonon scattering, where Umklapp scattering is neglected [19]. The second term at the righthand side stands for the non-locality. We suppose that the system is in quasi-stationarity, so that energy balances become 
$\nabla \cdot \boldsymbol{q}_{p \boldsymbol{h}}=0$

$\nabla \cdot \boldsymbol{q}_{\boldsymbol{e}}=\boldsymbol{E} \cdot \boldsymbol{I}$

$\nabla \cdot I=0$

Using Eqs. (8), Eqs. (7) become with quasi-stationarity

$\boldsymbol{q}_{p h}=-\lambda_{p h} \nabla T$

$\boldsymbol{q}_{\boldsymbol{e}}=-\lambda_{e} \nabla T+(\boldsymbol{E}-S \nabla T) \Pi \sigma_{e}$

$\boldsymbol{I}=\boldsymbol{E} \sigma_{e}-\mathrm{S} \sigma_{e} \nabla T$

From the above we can deduce that

$\boldsymbol{E}=\frac{\boldsymbol{I}}{\sigma_{e}}+\mathrm{S} \nabla T$

Inserting (10) in (9b), we find for a one-dimensional system (having only scalar values) and boundary conditions $T(z=0)=T_{c}$ and $T(z=L)=T_{h}$ (with $\left.\Delta T=T_{h}-T_{c}\right)$ :

$q_{p h}=\lambda_{p h} \frac{\Delta T}{L}$

$q_{e}=\lambda_{e} \frac{\Delta T}{L}+\Pi I$

Note by convention that the temperature gradient and electric field have the same direction, which is opposite to the one of the temperature difference, heat and current density fluxes. The thermoelectric efficiency is given by

$\eta_{t e}=\frac{P_{e}}{\dot{Q}}$

The electric power output is given by

$P_{e}=\boldsymbol{I} \cdot \int_{0}^{L} \boldsymbol{E} d y=I \Delta T S-I^{2} L \sigma_{e}^{-1}$

The total heat supplied, averaged over the thermoelectric element's length is given by

$\dot{Q}=\frac{1}{L} \int_{0}^{L} \boldsymbol{q} d y=\lambda_{p h} \frac{\Delta T}{L}+\lambda_{e} \frac{\Delta T}{L}+\Pi I$

Defining $\lambda_{t o t} \equiv \lambda_{p h}+\lambda_{e}$, the efficiency is finally given by

$\eta_{t e}=\frac{S I \Delta T-I^{2} L \sigma_{e}^{-1}}{\lambda_{t o t} \frac{\Delta T}{L}+\Pi I}$

The thermoelectric efficiency depends on an unknown current density, created by a temperature gradient. Defining $\Pi \equiv \mathrm{TS}$ (recalling that material properties are taken at $T=\mathrm{T}_{r e f}$ ), it is easy to see that an optimal current density, taking $\partial \eta_{t e} / \partial I=0$, can be found to be

$I_{o p t}=\frac{\lambda_{t o t} \Delta T(\sqrt{1+Z T}-1)}{S T L}$

so that a maximum thermoelectric efficiency can be found 
$\eta_{t e, \max }=\frac{\Delta T}{T_{t e, h}} \frac{Z T+2(1-\sqrt{1+Z T})}{Z T}$,

with

$Z T=T \frac{S^{2}}{\lambda_{t o t} / \sigma_{e}}$

the thermoelectric figure of merit. It should here kept in mind that $T_{h, t e}$ is the upper temperature of the thermoelectric device, which will be derived from the bottom temperature of the photovoltaic device $T_{p v, h}$ in Section 5.2. As such, we define $\Delta T \equiv T_{t e, h}-T_{t e, c}$, where $T_{t e, c}$ is the bottom temperature of the thermoelectric device. It can be seen that $\eta_{t e, \text { max }}$ can be increased by increasing $Z T$. In principle, these equations are developed and valid for both the $\mathrm{n}$ - and $\mathrm{p}$ leg of the thermoelectric element. We can define a thermoelectric efficiency for both legs altogether by defining in Eqs. (17)-(18)

$S=S_{p}-S_{n}$
$\frac{\lambda_{t o t}}{\sigma_{e}}=\left(\sqrt{\frac{\lambda_{p h, p}+\lambda_{e, p}}{\sigma_{e, p}}}+\sqrt{\frac{\lambda_{p h, n}+\lambda_{e, n}}{\sigma_{e, n}}}\right)^{2}$

where the subscripts $p$ and $n$ denote the p- and n-legs of the thermoelectric element, respectively. Since the thermoelectric legs are made out of nanocomposites, the aforementioned material properties are dependent on the matrix and nanoparticle ones.

\subsection{Material properties for the nanocomposite legs}

We present the development of the material properties for a certain nanocomposite leg $i$, with $i=p, n$. Since this is a heterogeneous medium, we use the well-known Effective Medium Approach (EMA) [20-22], which provides an analytical expression that homogenizes the nanocomposite medium. This approach, based on Maxwell's relation [20, 21] revisited by [22] and adapted here for our purposes, states [5] for the thermal conductivity

$\lambda_{c, i}=\lambda_{c, i}^{m} \frac{2 \lambda_{c, i}^{m}+\left(1+2 \alpha_{c, i}\right) \lambda_{c, i}^{p}+2 \varphi\left[\left(1-\alpha_{c, i}\right) \lambda_{c, i}^{p}-\lambda_{c, i}^{m}\right]}{2 \lambda_{c, i}^{m}+\left(1+2 \alpha_{c, i}\right) \lambda_{c, i}^{p}-\varphi\left[\left(1-\alpha_{c, i}\right) \lambda_{c, i}^{p}-\lambda_{c, i}^{m}\right]}$

This expression is valid for both the phonons $(c=p h)$ and electrons $(c=e)$. The analogy of the electron contribution with that of the phonon one is generally proposed throughout this development. From a physical point of view, the phonons and the electrons are considered as gas-like constituents, which behave as such in that they "flow" through the material lattice. We assume thusly that they also follow the same thermodynamic principles. The superscripts $m$ and $p$ concern the matrix and nanoparticle properties, $\varphi$ the nanoparticle volume fraction and $\alpha$ is a dimensionless parameter describing the nanoparticle-matrix interaction:

$\alpha_{c, i}=R_{c, i} \lambda_{c, i}^{m} / r_{p}$

Here, $r_{p}$ is the nanoparticle radius $\left(d_{p}=2 r_{p}\right.$ is the nanoparticle diameter) and the quantity $R$ is the thermal boundary resistance coefficient given by

$R_{c, i}=4 / C_{c, i}^{m} v_{c, i}^{m}+4 / C_{c, i}^{p} v_{c, i}^{p}$ 
where $C_{c}$ is the phonon specific heat and $v_{c}$ the phonon group velocity. The phonon thermal conductivity of the bulk matrix is given by the classical Boltzmann phonon expression:

$\lambda_{c, i}^{m}=\left.\frac{1}{3}\left(C_{c, i}^{m} v_{c, i}^{m} \Lambda_{c, i}^{m}\right)\right|_{T_{r e f}}$,

where $T_{r e f}$ is the reference temperature, say the room temperature. Within the matrix, the phonons experience phonon-phonon interactions and the mean free path is given by the Matthiessen rule:

$\frac{1}{\Lambda_{c, i}^{m}}=\frac{1}{\Lambda_{c, i, b}^{m}}+\frac{1}{\Lambda_{c, i, c o l l}^{m}}$

with $\Lambda_{c, i, b}^{m}$ designating the mean free path in the bulk matrix and $\Lambda_{c, i, c o l l}^{m}$ the supplementary contribution due to the interactions at the particle-matrix interface given by

$\Lambda_{c, i, \text { coll }}^{m}=\frac{4 r_{p}}{3 \varphi}$

The electric conductivity can be found through the electron thermal conductivity

$\sigma_{e, i}=\frac{\lambda_{e, i}}{L_{e} T}$

where $L_{e}$ is the Lorentz number and $T$ the absolute temperature. We note also that

$\sigma_{e, i}^{m}=\lambda_{e, i}^{m} / L_{e} T_{a m b}$

The Lorentz number is determined by

$L_{e}=\frac{\pi^{2}}{3}\left(\frac{k_{B}}{e_{c}}\right)^{2}$

where $k_{B}$ is Boltzmann's constant and $e_{c}$ the elementary charge. The Seebeck coefficient relates a temperature gradient with an electric current, albeit not directly. Indeed, the systems (7) and (9) show nicely that the thermal and electric conductivities precede the temperature gradient, while the Seebeck coefficient only precedes the temperature gradient in the form of a product with the electric conductivity. This motivates writing

$\left(S_{i} \sigma_{e, i}\right)=\left(S_{i}^{m} \sigma_{e, i}^{m}\right) \frac{2\left(s_{i}^{m} \sigma_{e, i}^{m}\right)+\left(1+2 \alpha_{e}\right)\left(s_{i}^{p} \sigma_{e, i}^{p}\right)+2 \varphi\left[\left(1-\alpha_{e}\right)\left(s_{i}^{p} \sigma_{e, i}^{p}\right)-\left(s_{i}^{m} \sigma_{e, i}^{m}\right)\right]}{2\left(s_{i}^{m} \sigma_{e, i}^{m}\right)+\left(1+2 \alpha_{e}\right)\left(s_{i}^{p} \sigma_{e, i}^{p}\right)-\varphi\left[\left(1-\alpha_{e}\right)\left(s_{i}^{p} \sigma_{e, i}^{p}\right)-\left(s_{i}^{m} \sigma_{e, i}^{m}\right)\right]}$.

where $S_{i}^{m}$ is the bulk value of the Seebeck coefficient of the matrix. The overall Seebeck coefficient of one leg can then be easily obtained a posteriori, by defining

$S_{i}=\left(S_{i} \sigma_{e, i}\right) / \sigma_{e, i}$,

The thermoelectric model is complete when we find an expression for the phonon and electron thermoelectric conductivity and the Seebeck coefficient of the nanoparticles, i.e. $\lambda_{p h, i}^{p}, \lambda_{e, i}^{p}$ and $S_{i}^{p}$. In order to find these, we have to take into account size effects at nanoscale. For this, we 
use EIT. The non-local effects that are introduced by EIT (in the framework of this paper) for the phonon contribution, apply also for the electron contribution of the thermal conductivity. As the non-local effects also apply for the electron contribution, the electrical conductivity can be treated in the same way in the same framework as well as the product of the Seebeck coefficient and the electric conductivity. The phonon thermal conductivity at nanoscale is given by

$\lambda_{c, i}^{p}=\lambda_{c, i}^{p, 0} f\left(K n_{c}\right)$

wherein $\lambda_{c, i}^{p, 0}$ is the value of the phonon/electron thermal conductivity for the bulk material from which the nanoparticle is made of, the 0 indicating a reference value:

$\lambda_{c, i}^{p, 0}=\left.\frac{1}{3}\left(C_{c, i}^{p} v_{c, i}^{p} \Lambda_{c, i}^{p}\right)\right|_{T_{r e f}}$

The Knudsen number is given by

$K n_{c}=\Lambda_{c, i}^{p} / r_{p}$

We note that

$\sigma_{e, i}^{p}=\lambda_{c, i}^{p} / L_{e} T$

We have shown in a previous work [6] that

$S_{i}^{p} \sim\left(\sigma_{e, i}^{p}\right)^{-1}$

The only quantity still to be found is $f\left(K n_{c}\right)$ a correction factor, taking into account the dimension of the nanoparticles. We derive this correction factor by means of EIT in the following section.

\section{Nanoscale material properties proposed by EIT}

Let us consider for the purposes of this work a rigid and isotropic body (with constant density), which is crossed by a heat flux $\boldsymbol{q}$ and an electric flux $\boldsymbol{I}$. Then, the relevant conserved variables are the internal energy $u$ (or the temperature $T$ ) and the electric charge density $\varrho_{e}$, whereas the energy flux (here the heat flux vector $\boldsymbol{q}$ ) and the electric flux (here the electric current density $\boldsymbol{I})$ are the non-conserved flux variables so that the space of state variables is $\boldsymbol{V}=\left(u, \varrho_{e}, \boldsymbol{q}, \boldsymbol{I}\right)$. However, in more complex materials like in nanomaterials, it is necessary to introduce fluxes of higher order, as will be shown later on. The corner stone of EIT is to assume the existence of an entropy function $s(\boldsymbol{V})$ [13], depending on the whole set $\boldsymbol{V}$ of variables: here $s=$ $s\left(u, \varrho_{e}, \boldsymbol{q}, \boldsymbol{I}\right)$, or in terms of time derivatives,

$d_{t} s\left(u, \varrho_{e}, \boldsymbol{q}, \boldsymbol{I}\right)=\frac{\partial s}{\partial u} d_{t} u+\frac{\partial s}{\partial \boldsymbol{q}} \cdot d_{t} \boldsymbol{q}+\frac{\partial s}{\partial \varrho_{e}} d_{t} \varrho_{e}+\frac{\partial s}{\partial \boldsymbol{I}} \cdot d_{t} \boldsymbol{I}$

wherein $u$ and $s$ are measured per unit volume and a dot stands for the scalar product. The symbol $d_{t}$ designates the time derivative which is indifferently the material or the partial time derivative as the system is, respectively, in motion or at rest. Here, $s$ is assumed to be a concave 
function of the variables in order to guarantee stability of the equilibrium state obeying at the same time a general time-evolution equation of the form

$d_{t} s=-\nabla \cdot J^{s}+\sigma^{s} \quad\left(\sigma^{s} \geq 0\right)$,

whose rate of production per unit volume $\sigma^{S}$ (in short, the entropy production) is positive definite to satisfy the second principle of thermodynamics, while the quantity $\boldsymbol{J}^{S}$ is the entropy flux. Let us define the local non-equilibrium temperature by $T^{-1}(u)=\partial s / \partial u$ (fundamental thermodynamic relation at constant volume) and define $\partial s / \partial \varrho_{e}=-T^{-1} \mu_{e}$, where $\mu_{e}$ is the chemical potential of electrons. Let us also select the simplified constitutive equations for $\partial s / \partial \boldsymbol{q}$ and $\partial s / \partial \boldsymbol{I}$ as given by $\partial s / \partial \boldsymbol{q}=-\left(\gamma_{\boldsymbol{q}}^{\boldsymbol{q}} \boldsymbol{q}+\gamma_{\boldsymbol{q}}^{\boldsymbol{I}} \boldsymbol{I}\right)$ and $\partial s / \partial \boldsymbol{I}=-\left(\gamma_{\boldsymbol{I}}^{\boldsymbol{q}} \boldsymbol{q}+\gamma_{\boldsymbol{I}}^{\boldsymbol{I}} \boldsymbol{I}\right)$, respectively. There, $\gamma^{q}(T)$ and $\gamma^{I}(T)$ are material coefficients depending generally on the temperature, where the subscript indicates to what the entropy is derived. These material coefficients are positive definite so that the property that $s$ is maximum at equilibrium is met. With these conditions, expression (37), referred to as the Gibbs equation, can be written as

$$
d_{t} s\left(u, \varrho_{e}, \boldsymbol{q}, \boldsymbol{I}\right)=T^{-1} d_{t} u-\left(\gamma_{\boldsymbol{q}, 1}^{\boldsymbol{q}} \boldsymbol{q}+\gamma_{\boldsymbol{q}, 1}^{\boldsymbol{I}} \boldsymbol{I}\right) \cdot d_{t} \boldsymbol{q}-\mu_{e} T^{-1} d_{t} \varrho_{e}-\left(\gamma_{\boldsymbol{I}, 1}^{\boldsymbol{q}} \boldsymbol{q}+\gamma_{\boldsymbol{I}, 1}^{\boldsymbol{I}} \boldsymbol{I}\right) \cdot d_{t} \boldsymbol{I}
$$

wherein $\gamma_{j, 1}^{i}(i=\boldsymbol{q}, \boldsymbol{I}$ and independently $\boldsymbol{j}=\boldsymbol{q}, \boldsymbol{I})$ are phenomenological crossing coefficients identified later on. However, expression (39) does not account for non-local effects. These nonlocal effects are elegantly introduced in the framework of EIT by appealing to a hierarchy of fluxes $\boldsymbol{Q}^{(1)}, \boldsymbol{Q}^{(2)}, \ldots, \boldsymbol{Q}^{(n)}$ with $\boldsymbol{Q}^{(1)}$ identical to the heat flux vector $\boldsymbol{q}, \boldsymbol{Q}^{(2)}$ (a tensor of rank two) is the flux of $\boldsymbol{q}, \boldsymbol{Q}^{(3)}$ the flux of $\boldsymbol{Q}^{(2)}$ and so on. The same is done for the electric current density, introducing the fluxes $\boldsymbol{I}^{(1)}\left(\boldsymbol{I} \equiv \boldsymbol{I}^{(1)}\right), \boldsymbol{I}^{(2)}, \ldots, \boldsymbol{I}^{(n)}$. Up to the $\mathrm{n}^{\text {th }}$-order flux, the Gibbs equation generalizing relation (39) becomes

$$
\begin{aligned}
& d_{t} s\left(u, \varrho_{e}, \boldsymbol{q}, \boldsymbol{Q}^{(2)}, \ldots, \boldsymbol{Q}^{(n)}, \boldsymbol{I}, \boldsymbol{I}^{(2)}, \ldots, \boldsymbol{I}^{(n)}\right)=T^{-1} d_{t} u-\mu_{e} T^{-1} d_{t} \varrho_{e}-\left(\gamma_{\boldsymbol{q}, 1}^{\boldsymbol{q}} \boldsymbol{q}+\gamma_{\boldsymbol{q}, 1}^{\boldsymbol{I}} \boldsymbol{I}\right) \cdot d_{t} \boldsymbol{q}- \\
& \left(\gamma_{\boldsymbol{q}, 2}^{\boldsymbol{q}} \boldsymbol{Q}^{(2)}+\gamma_{\boldsymbol{q}, 2}^{\boldsymbol{I}} \boldsymbol{I}^{(2)}\right) \otimes d_{t} \boldsymbol{Q}^{(2)}-\cdots-\left(\gamma_{\boldsymbol{q}, n}^{\boldsymbol{q}} \boldsymbol{Q}^{(n)}+\gamma_{\boldsymbol{q}, n}^{\boldsymbol{I}} \boldsymbol{I}^{(n)}\right) \otimes d_{t} \boldsymbol{Q}^{(n)}-\left(\gamma_{\boldsymbol{I}, 1}^{\boldsymbol{q}} \boldsymbol{q}+\gamma_{\boldsymbol{I}, 1}^{\boldsymbol{I}} \boldsymbol{I}\right) \cdot d_{t} \boldsymbol{I}- \\
& \left(\gamma_{\boldsymbol{I}, 2}^{\boldsymbol{q}} \boldsymbol{Q}^{(2)}+\gamma_{\boldsymbol{I}, 2}^{\boldsymbol{I}} \boldsymbol{I}^{(2)}\right) \otimes d_{t} \boldsymbol{I}^{(2)}-\cdots-\left(\gamma_{\boldsymbol{I}, n}^{\boldsymbol{q}} \boldsymbol{Q}^{(\boldsymbol{n})}+\gamma_{\boldsymbol{I}, n}^{\boldsymbol{I}} \boldsymbol{I}^{(n)}\right) \otimes d_{t} \boldsymbol{I}^{(n)}
\end{aligned}
$$

wherein the symbol $\otimes$ denotes the inner product of the corresponding tensors. The subsequent step is the formulation of the entropy flux $\boldsymbol{J}^{\boldsymbol{s}}$. It is natural to expect that it is not simply given by the classical expression $\left(T^{-1} \boldsymbol{q}-\mu_{e} T^{-1} \boldsymbol{I}\right)$, but that it will depend on higher order fluxes in a similar way as for the generalized Gibbs equation, so that it is assumed that

$\boldsymbol{J}^{S}=T^{-1} \boldsymbol{q}-\mu_{e} T^{-1} \boldsymbol{I}+\Gamma_{\boldsymbol{q}, 1} \boldsymbol{Q}^{(2)} \cdot \boldsymbol{q}+\cdots+\Gamma_{\boldsymbol{q}, n-1} \boldsymbol{Q}^{(n)} \otimes \boldsymbol{Q}^{(n-1)}-\Gamma_{\boldsymbol{I}, 1} \boldsymbol{I}^{(2)} \cdot \boldsymbol{I}-\cdots-$ $\Gamma_{I, n-1} I^{(2)} \cdot \boldsymbol{I}$,

where the $\Gamma$ 's are material coefficients in analogy to the $\gamma$ 's. The next step is the derivation of the rate of entropy production per unit volume $\sigma^{\mathrm{S}}$ which, referring to (38), is given by

$$
\sigma^{s}=d_{t} s+\nabla \cdot \boldsymbol{J}^{s} \geq \mathbf{0}
$$

After substituting in (42) the expressions of $d_{t} s$ and $\boldsymbol{J}^{\boldsymbol{s}}$ from (40) and (41), respectively, and eliminating $d_{t} u$ via the energy conservation law for rigid heat conductors $\left(d_{t} u=-\nabla \cdot \boldsymbol{q}+\boldsymbol{I}\right.$. $\boldsymbol{E})$ and $d_{t} \varrho_{e}$ via the continuity law for electric charge $\left(d_{t} \varrho_{e}=-\nabla \cdot \boldsymbol{I}\right)$, one obtains 


$$
\begin{aligned}
& \sigma^{S}=-\left(-\nabla T^{-1}+\gamma_{\boldsymbol{q}, 1}^{\boldsymbol{q}} d_{t} \boldsymbol{q}+\gamma_{\boldsymbol{I}, 1}^{\boldsymbol{q}} d_{t} \boldsymbol{I}-\Gamma_{\boldsymbol{q}, 1} \nabla \cdot \boldsymbol{Q}^{(2)}\right) \cdot \boldsymbol{q}-\left(-T^{-1} \boldsymbol{E}+\nabla \mu_{e} T^{-1}+\gamma_{\boldsymbol{q}, 1}^{\boldsymbol{I}} d_{t} \boldsymbol{q}+\right. \\
& \left.\gamma_{\boldsymbol{I}, 1}^{\boldsymbol{I}} d_{t} \boldsymbol{I}-\Gamma_{\boldsymbol{I}, 1} \nabla \cdot \boldsymbol{Q}^{(2)}\right) \cdot \boldsymbol{I}-\cdots-\sum_{n=2}^{N} \boldsymbol{Q}^{(n)} \otimes\left(\gamma_{\boldsymbol{q}, n}^{\boldsymbol{q}} d_{t} \boldsymbol{Q}^{(n)}+\gamma_{\boldsymbol{I}, n}^{\boldsymbol{q}} d_{t} \boldsymbol{I}^{(n)}-\Gamma_{\boldsymbol{q}, n} \nabla \cdot \boldsymbol{Q}^{(n+1)}-\right. \\
& \left.\Gamma_{\boldsymbol{q}, n-1} \nabla \boldsymbol{Q}^{(n-1)}\right)-\cdots-\sum_{n=2}^{N} \boldsymbol{I}^{(n)} \otimes\left(\gamma_{\boldsymbol{q}, n}^{\boldsymbol{I}} d_{t} \boldsymbol{Q}^{(n)}+\gamma_{\boldsymbol{I}, n}^{\boldsymbol{I}} d_{t} \boldsymbol{I}^{(n)}-\Gamma_{I, n} \nabla \cdot \boldsymbol{I}^{(n+1)}-\right. \\
& \left.\Gamma_{\boldsymbol{I}, n-1} \nabla \boldsymbol{I}^{(n-1)}\right) \geq 0
\end{aligned}
$$

The expression for $\sigma^{S}$ is a bilinear relationship in the fluxes $\boldsymbol{q}$ and $\boldsymbol{I}$ and the subsequent higher order of fluxes $\boldsymbol{Q}^{(n)}$ and $\boldsymbol{I}^{(n)}$. The quantities represented by the terms between the parentheses are usually called the thermodynamic forces $\boldsymbol{X}_{i}$. The simplest way to guarantee the positiveness of the entropy production $\sigma^{s}$ is to assume a linear flux-force relation of the forms $\boldsymbol{Q}^{(n)}=\beta_{\boldsymbol{q}} \boldsymbol{X}_{\boldsymbol{q}}$ and $\boldsymbol{I}^{(n)}=\beta_{\boldsymbol{I}} \boldsymbol{X}_{\boldsymbol{I}}(n=1,2,3, \ldots, N)$, where the $\beta_{i}$ 's are phenomenological coefficients. As such, we obtain

$$
\begin{aligned}
& \nabla T^{-1}-\gamma_{\boldsymbol{q}, 1}^{\boldsymbol{q}} d_{t} \boldsymbol{q}-\gamma_{\boldsymbol{I}, 1}^{\boldsymbol{q}} d_{t} \boldsymbol{I}+\Gamma_{\boldsymbol{q}, 1} \nabla \cdot \boldsymbol{Q}^{(2)}=\chi_{\boldsymbol{q}, 1} \boldsymbol{q}, \\
& T^{-1} \boldsymbol{E}-\nabla \mu_{e} T^{-1}-\gamma_{\boldsymbol{q}, 1}^{\boldsymbol{I}} d_{t} \boldsymbol{q}-\gamma_{\boldsymbol{I}, 1}^{\boldsymbol{I}} d_{t} \boldsymbol{I}+\Gamma_{\boldsymbol{I}, 1} \nabla \cdot \boldsymbol{I}^{(2)}=\chi_{\boldsymbol{I}, 1} \boldsymbol{I} \\
& \Gamma_{q, n-1} \nabla \boldsymbol{Q}^{(n-1)}-\gamma_{\boldsymbol{q}, n}^{\boldsymbol{q}} d_{t} \boldsymbol{Q}^{(n)}-\gamma_{\boldsymbol{I}, n}^{\boldsymbol{q}} d_{t} \boldsymbol{I}^{(n)}+\Gamma_{\boldsymbol{q}, n} \nabla \cdot \boldsymbol{Q}^{(n+1)}=\chi_{\boldsymbol{q}, n} \boldsymbol{Q}^{(n)},(n=2,3, \ldots, N), \\
& \Gamma_{I, n-1} \nabla \boldsymbol{I}-\gamma_{\boldsymbol{q}, n}^{\boldsymbol{I}} d_{t} \boldsymbol{Q}^{(n)}-\gamma_{\boldsymbol{I}, n}^{\boldsymbol{I}} d_{t} \boldsymbol{I}^{(n)}+\Gamma_{\boldsymbol{I}, n} \nabla \cdot \boldsymbol{I}^{(n+1)}=\chi_{\boldsymbol{I}, n} \boldsymbol{I}^{(n)}, \quad(n=2,3, \ldots, N),
\end{aligned}
$$

compatible with positive entropy production at the condition that $\chi_{\boldsymbol{q}, 1} \geq 0, \chi_{I, 1} \geq 0, \chi_{\boldsymbol{q}, n} \geq 0$ and $\chi_{I, n} \geq 0$. In (44)-(45), $d_{t}$ stands for the partial time derivative, if we assume the material to be at rest. Also, $\gamma_{i}, \Gamma_{i}$ and $\chi_{i}$ are phenomenological coefficients related to the relaxation times, correlation lengths and transport coefficients, respectively. Eq. (44a) reduces to the wellknown Cattaneo's law [23] when the terms $\nabla \cdot \boldsymbol{Q}^{(2)}$ and $d_{t} \boldsymbol{I}$ are omitted. Furthermore, if the term $d_{t} \boldsymbol{q}$ is neglected, we obtain Fourier's law. Note that Eq. (44b) also reduces to a Cattaneolike law when one omits $\nabla \cdot \boldsymbol{I}^{(2)}$ and $d_{t} \boldsymbol{q}$. Ohm's law is obtained when furthermore the term $d_{t} \boldsymbol{I}$ is neglected. In order to simplify the following procedure, we focus on only the heat flux. We now consider an infinite number of flux variables $(N \rightarrow \infty)$ and apply the spatial Fourier transform

$$
\widehat{\boldsymbol{q}}(\boldsymbol{k}, t)=\int_{-\infty}^{+\infty} \boldsymbol{q}(\boldsymbol{r}, t) e^{-i \boldsymbol{k} \cdot \boldsymbol{r}} d \boldsymbol{r}
$$

to Eqs. (44a) and (45a) neglecting for simplification the cross terms $d_{t} \boldsymbol{I}^{(n)}(n=1,2,3, \ldots)$, with $\widehat{\boldsymbol{q}}$ the Fourier transform of $\boldsymbol{q}, \boldsymbol{r}$ the spatial variable, $t$ the time and $\boldsymbol{k}$ the wavenumber vector. This procedure results into obtaining the following time-evolution equation for the heat flux:

$$
\bar{\tau}(\boldsymbol{k}) \partial_{t} \widehat{\boldsymbol{q}}(\boldsymbol{k}, t)+\widehat{\boldsymbol{q}}(\boldsymbol{k}, t)=-i \boldsymbol{k} \lambda_{c, i}^{p}(\boldsymbol{k}) \widehat{T}(\boldsymbol{k}, t)
$$

where $\bar{\tau}(\boldsymbol{k})=\gamma_{1} / \mu_{1}$ designates a renormalized relaxation time depending generally on $\boldsymbol{k}$. $\lambda_{c, i}^{p}(\boldsymbol{k})$ is given by the continued-fraction for the $\boldsymbol{k}$-dependent effective thermal conductivity:

$$
\lambda_{c, i}^{p}(\boldsymbol{k})=\frac{\lambda_{c, i}^{p, 0}}{1+\frac{\boldsymbol{k}^{2} l_{1}^{2}}{1+\frac{\boldsymbol{k}^{2} l_{2}^{2}}{1+\boldsymbol{k}^{2} l_{3}^{2}}}}
$$


with $\lambda_{c, i}^{p, 0}$ the classical bulk thermal conductivity, given by Eq. (33), independent of the dimension of the system, $l_{n}$ is the correlation length of order $n$ defined by $l_{n}^{2}=\gamma_{n}^{2} /\left(\mu_{n} \mu_{n+1}\right)$. Here, it is assumed that the relaxation times $\tau_{n}(n>1)$ corresponding to higher order fluxes are negligible with respect to $\tau_{1}$, which is a hypothesis generally admitted in kinetic theories. In the present problem, there is only one dimension (the nanoparticle radius), so that it is natural to define $k \equiv 2 \pi / r_{p}$. The correlation lengths selected as $l_{n}^{2}=a_{n+1} l^{2}$, with $a_{n}=n^{2} /\left(4 n^{2}-\right.$ $1)$ and $l$ identified as the mean free path independently of the order of approximation. This is a rather natural choice for phonons as shown by Dreyer and Struchtrup [24], which we, as mentioned earlier, apply for electrons as well. With these results in mind, the continued fraction (48) reduces, within the asymptotic limit $N \rightarrow \infty$ (see for mathematical formulation Ref. [25]) to the following expression for $\lambda_{c, i}^{p}$ :

$\lambda_{c, i}^{p}=\frac{3 \lambda_{c, i}^{p, 0}}{4 \pi^{2} K n_{c}^{2}}\left[\frac{2 \pi K n_{c}}{\arctan \left(2 \pi K n_{c}\right)}-1\right]$

with $K n_{c}$ given by Eq. (34). The same can be found for the electrical conductivity in analogy with the thermal conductivity. Hereby, the correlation lengths are only associated with the mean free path of the electrons. The final result is

$\sigma_{e, i}^{p}=\frac{3 \sigma_{e, i}^{p, 0}}{4 \pi^{2} K n_{e}^{2}}\left[\frac{2 \pi K n_{e}}{\arctan \left(2 \pi K n_{e}\right)}-1\right]$

with $K n_{e}$ given by Eq. (34) with $c=e$. We should note that the relation between (49a) and (49b) is still valid by Eq. (35).

\section{Opto-electric model for the photovoltaic device \\ 4.1 Basic considerations}

For the photovoltaic device we use the semiconductor equations for electron transport

$\nabla \cdot(\varepsilon \nabla V)=-e_{C}\left(p-n+N_{D}-N_{A}\right)$

$\nabla \cdot \boldsymbol{I}_{p}=e_{C}(G-R)$

$\nabla \cdot I_{n}=-e_{C}(G-R)$

$\boldsymbol{I}_{P}=-\mu_{p}\left(e_{C} p \nabla V+k_{B} T \nabla p\right)$

$\boldsymbol{I}_{n}=-\mu_{n}\left(e_{C} n \nabla V-k_{B} T \nabla n\right)$

with $\varepsilon$ the dielectric constant of the semiconductor $\left(\varepsilon \equiv \varepsilon_{r} \varepsilon_{0}\right.$, with $\varepsilon_{r}$ the relative permittivity with respect to the vacuum permittivity $\left.\varepsilon_{0}\right), V$ the electric potential, $e_{C}$ the electron charge, $p$ and $n$ the hole and electron carrier concentrations, respectively, $N_{D}$ and $N_{A}$ the donor and acceptor doping concentrations, respectively, $\boldsymbol{I}_{p}$ and $\boldsymbol{I}_{n}$ the hole and electron current densities, respectively, $G$ and $R$ the generation and recombination rates, respectively. The Eqs. (50)-(54) represent Poisson's equation relating the electrical potential to the space charge density, the continuity equations for holes and electrons and the hole and electron current densities, respectively. Note that in Poisson's equation $\nabla \cdot(\varepsilon \nabla V)=\nabla \cdot(\varepsilon \mathbf{E})$, where $\mathbf{E}$ is the electric field.

The generation rate $G$ is given by

$$
G=\int_{k_{\min }}^{h c / E_{g}} \alpha(k) e^{-\alpha(k) z} S_{A M 1.5}(k) \mathrm{d} k
$$


where $h$ is Planck's constant, $c$ the speed of light, $E_{g}$ the band-gap energy, $\alpha$ the absorption coefficient, $k$ the photon wavelength, $z$ the space coordinate and $S_{A M 1.5}$ is defined as the standard number of incident photons per surface per wavelength: $S_{A M 1.5}(k) \equiv I_{A M 1.5} /$ $E_{\text {photon }}(k)$, where $E_{\text {photon }}$ is the photon energy. $I_{A M 1.5}$ is defined as the incident photon energy per surface per wavelength, where the subscript stands for the air mass number. In (55), $k_{\text {min }}$ is the minimum photon wavenlength for solar irradiance, being $400 \mathrm{~nm}$ [26]. Note that $k_{\max }$, needed later on, is the maximum one, being $1050 \mathrm{~nm}$ [26]. The particularity of $S_{A M 1.5}(k)$ is that it depends on the wavelength of the different "light spectra", i.e. blue light has a lower wavelength than for instance red light and has thus higher photon energy. Therefore, we have to integrate over the whole spectrum from $k_{\min }$ to $h c / E_{g}$ in Eq. (55). We define $I_{A M 1.5} \equiv$ $\partial P_{S} / \partial k$, where $P_{S}$ is the solar energy per surface for a given wavelength. There is no formulation for $I_{A M 1.5}$. We, therefore, take an average integrated value over the photon wavelengths: $\left\langle I_{A M 1.5}\right\rangle \equiv \int_{k_{\min }}^{h c / E_{g}} I_{A M 1.5} d k=\int_{k_{\min }}^{h c / E_{g}} \frac{\partial P_{s}}{\partial k} d k \approx P_{S}$. Typically, $P_{S}$ is of the order of magnitude of $1000 \mathrm{~W} / \mathrm{m}^{2}$. The absorption coefficient is also averaged over the wavelength: $\langle\alpha\rangle \equiv$ $\int_{k_{\min }}^{h c / E_{g}} \alpha(k) d k$. We also take an averaged value for the thickness dependence of $G$,

$\left\langle\alpha e^{-\alpha z}\right\rangle=\frac{1-e^{-\alpha \delta}}{\delta}$

We average also the photon energy $E_{\text {photon }}=\frac{h c}{2 \pi k}$

$\left\langle E_{\text {photon }}\right\rangle=\frac{h c}{2 \pi\left(\frac{h c}{E_{g}}-k_{\text {min }}\right)} \ln \left(\frac{\frac{h c}{E_{g}}}{k_{\text {min }}}\right)$

Finally, we have

$G \approx P_{S} \frac{1-e^{-\alpha \delta}}{\delta} \frac{2 \pi\left(\frac{h c}{E_{g}}-k_{\min }\right)}{h c} \frac{1}{\ln \left(\frac{\frac{h c}{E_{g}}}{k_{\min }}\right)}$

This form has also been suggested in [27]. Finally, note that thusly we do not take $G$ as a given constant value, often done in analytical developments, but dependent on the device's thickness. The recombination rate $R$ is given by

$R=R_{S R H}+R_{A u g}+g R_{\text {surf }}$

where

$R_{S R H}=\frac{p n-n_{i e}^{2}}{\tau_{n}\left(p+n_{i e}\right)+\tau_{p}\left(n+n_{i e}\right)}$

$R_{\text {Aug }}=\left(B_{p} p+B_{n} n\right)\left(p n-n_{i e}^{2}\right)$

$R_{\text {surf }}=\frac{p_{s} n_{s}-n_{\text {ies }}^{2}}{\tau_{n s f}\left(p_{s}+n_{\text {ies }}\right)+\tau_{p s f}\left(n_{s}+n_{\text {ies }}\right)}$

and in (59)-(60c), we used 
$g \equiv \frac{N}{N_{S}}$

$n_{i e}=\sqrt{N_{C} N_{V}} e^{-\frac{E g}{2 k_{B} T}}$

In (59)-(62), $R_{S R H}, R_{A u g}$ and $R_{\text {surf }}$ are the so-called Shockley-Read-Hall (SRH), Auger recombination and surface recombination rates, respectively, $\tau_{p}$ and $\tau_{n}$ the minority hole and electron SRH lifetimes, $W_{p}$ and $W_{n}$ the $\mathrm{p}$ - and $\mathrm{n}$-sides of the quasi-neutral layers (explained later in the paper), $S_{r p}$ and $S_{r n}$ the minority hole and electron surface recombination velocities, $n_{i e}$ the intrinsic carrier concentration in the recombination sites, $B_{p}$ and $B_{n}$ the hole and electron Auger recombination coefficients, respectively, $\tau_{p s f}$ and $\tau_{n s f}$ the hole and electron surface recombination lifetimes, respectively, and $N_{C}$ and $N_{V}$ the effective density of states for the conduction and valence bands, respectively. Note that in (59) and (61), $g$ is a proportionality factor, being defined here as the ratio of a bulk (equilibrium) carrier concentration $(N=$ $\left.p, n, n_{i e}\right)$ and a surface (equilibrium) carrier concentration $\left(N_{s}=p_{s}, n_{s}, n_{i e s}\right)$. Radiative recombination is neglected [28].

In order to solve for all these equations, we need to make first a difference between the depletion region and the quasi-neutral regions. We will start with solving for the depletion region in Section 4.2. In Section 4.3 we treat the quasi-neutral regions. The findings are synthesized in Section 4.4 to obtain the photovoltaic efficiency.

\subsection{Depletion region}

We make certain assumptions in order to solve the equations analytically:

- Depletion approximation: the electric field is confined to the junction region (depletion region) and there is none in the quasi-neutral regions;

- The number of free carriers in the depletion region is small, assuming that the electric field sweeps them out of the depletion region quickly. This means that we do not consider the transport equation for the carrier concentrations and that we can neglect recombination. We consider generation in the depletion region;

- Abrupt or step doping profile, where all dopants are ionized: the acceptor and donor doping concentrations are constant in their respective regions;

- One-dimensional system;

- We define $z=0$ as the point of the p-n junction, $z=-z_{p}$ the p-side of the depletion region and $z=z_{n}$ the n-side of the depletion region;

- Constant electric permittivity at each side of the p-n junction.

The system to solve becomes

$\frac{d^{2} V}{d z^{2}}=\left\{\begin{array}{lr}\frac{e_{C}}{\varepsilon_{p}} N_{A}, & -z_{p} \leq z<0 \\ -\frac{e_{C}}{\varepsilon_{n}} N_{D}, & 0 \leq z<z_{n}\end{array}\right.$

$\frac{d I_{p}}{d z}=e_{C} G$

$\frac{d I_{n}}{d z}=-e_{C} G$

The subscripts of the relative permittivity denote that it concerns either the $\mathrm{p}$-side or the $\mathrm{n}$-side of the depletion region. So we take into account the difference in permittivity of the two materials, which is not often done. Since we have assumed that there is no electric field outside the depletion region, we have as boundary conditions $\frac{d V}{d z}=0$ at $z=-z_{p}$ and $z=z_{n}$. One is 
usually interested in the potential difference across the junction. We define here the voltage on the p-side as $V_{0}$ (applied voltage in case of a diode or induced voltage in case of a solar cell), at $z=-z_{p}$. At the n-p interface the potential should be equal on both sides, so that $V_{p}=V_{n}$ at $z=$ 0 (where the subscripts $p$ and $n$ indicate the $\mathrm{p}$ - and n-sides of the interface, respectively). The electric potential is then given by

$V(z)=\left\{\begin{array}{lr}V_{0}+\frac{e_{C} N_{A}}{2 \varepsilon_{p}}\left(z+z_{p}\right)^{2}, & -z_{p} \leq z<0 \\ V_{0}+\frac{e_{C} N_{A}}{2 \varepsilon_{p}} z_{p}^{2}+\frac{e_{C} N_{D}}{\varepsilon_{n}}\left(z_{n}-\frac{z}{2}\right) z, & 0 \leq z<z_{n}\end{array}\right.$

It is now necessary to know $z_{p}$ and $z_{n}$. At the interface $z=0$, we assume displacement vector $(\boldsymbol{D}=\varepsilon \boldsymbol{E})$ continuity. So $\varepsilon_{p} \frac{d V_{p}}{d z}=\varepsilon_{n} \frac{d V_{n}}{d z}$ at $z=0$. This gives

$N_{A} z_{p}=N_{D} z_{n}$

The maximum voltage across the junction is at $z=z_{n}$ being

$V\left(z_{n}\right)=V_{0}+\frac{e_{C}}{2}\left(\frac{N_{A} z_{p}^{2}}{\varepsilon_{p}}+\frac{N_{D} z_{n}^{2}}{\varepsilon_{n}}\right)$

This voltage is also equal to the built-in voltage (which can be found from the difference in Fermi-levels between the $\mathrm{p}$ - and n-side materials) across the $\mathrm{p}$-n junction, $V_{D}$, so that

$V_{D}=V_{0}+\frac{e_{C}}{2}\left(\frac{N_{A} z_{p}^{2}}{\varepsilon_{p}}+\frac{N_{D} z_{n}^{2}}{\varepsilon_{n}}\right)$

with

$V_{D}=\frac{k_{B} T}{e_{C}} \ln \left(\frac{N_{A} N_{D}}{n_{i e}^{2}}\right)$

We can finally find that

$$
\begin{aligned}
& z_{p}=\sqrt{\frac{2\left(V_{D}-V_{0}\right)}{e_{C}} \frac{N_{D}}{N_{A}\left(\frac{N_{A}}{\varepsilon_{n}}+\frac{N_{D}}{\varepsilon_{p}}\right)}} \\
& z_{n}=\sqrt{\frac{2\left(V_{D}-V_{0}\right)}{e_{C}} \frac{N_{A}}{N_{D}\left(\frac{N_{A}}{\varepsilon_{n}}+\frac{N_{D}}{\varepsilon_{p}}\right)}}
\end{aligned}
$$

The total width of the depletion region is then

$$
W_{d}=z_{p}+z_{n}=\sqrt{\frac{2\left(V_{D}-V_{0}\right)}{e_{C}}\left(\frac{\varepsilon_{n}}{N_{A}}+\frac{\varepsilon_{p}}{N_{D}}\right)}
$$


which shows that the depletion region depends on the material properties of both the $\mathrm{p}$ - and nside ones as well as on an applied/induced voltage. Note also that

$z_{p}=W_{d} \frac{\frac{N_{D}}{\varepsilon_{n}}}{\frac{N_{A}}{\varepsilon_{p}}+\frac{N_{D}}{\varepsilon_{n}}}$
$z_{n}=W_{d} \frac{\frac{N_{A}}{\varepsilon_{p}}}{\frac{N_{A}}{\varepsilon_{p}}+\frac{N_{D}}{\varepsilon_{n}}}$

Now, under solar illumination, there is also generation in the depletion region, as shown in Eqs. (64)-(65). Integrating between the depletion borders gives $I_{n}\left(z=z_{n}\right)-I_{n}\left(z=-z_{p}\right)=$ $-e_{C}\left(C_{G p} z_{p}+C_{G n} z_{n}\right)$, so that we define a surplus hole current density through the depletion region $\Delta I_{p, d}$ :

$\Delta I_{p, d}=e_{C}\left(C_{G p} z_{p}+C_{G n} z_{n}\right)$

Note that the same can be done for the electron current density $\Delta I_{n, d}$ giving

$\Delta I_{n, d}=-e_{C}\left(C_{G p} z_{p}+C_{G n} z_{n}\right)$

It can be easily seen that the net current density through the depletion region is constant.

\subsection{Quasi-neutral regions}

We continue now with the quasi-neutral regions. Having said that there is no electrical field in the quasi-neutral regions, we can omit Poisson's equation from this development (the electric potential in the $\mathrm{p}$ - and $\mathrm{n}$-sides of the quasi-neutral regions are constant and equal to the ones at the respective depletion region borders). The drift terms in the transport equations also becomes zero. The system to solve becomes

$\frac{d I_{p}}{d z}=e_{C}(G-R)$

$\frac{d I_{n}}{d z}=-e_{C}(G-R)$

$I_{p}=-\mu_{p} k_{B} T \frac{d p}{d z}$

$I_{n}=\mu_{n} k_{B} T \frac{d n}{d z}$

We can rewrite this set of equations as

$$
\begin{aligned}
& k_{B} T \frac{\mu_{p}}{e_{C}} \frac{d^{2} p}{d z^{2}}+(G-R)=0 \\
& k_{B} T \frac{\mu_{n}}{e_{C}} \frac{d^{2} n}{d z^{2}}+(G-R)=0
\end{aligned}
$$

For the generation rate, we have established an averaged expression, depending now only on the total thickness of the device so that we define $G \equiv C_{G}$. We define also an average generation rate for the n-side 


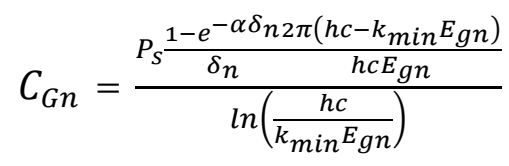

We can also define an average generation rate for the p-side, but with the assumption that only a fraction $e^{-\alpha \delta_{n}}$ remains, so that

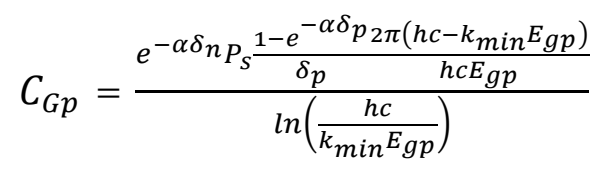

As for $R$, we make the assumption of low injection, where it is assumed that the majority carrier concentrations are unperturbed throughout the quasi-neutral regions. This means that $p_{p} \gg n_{p}$ and $p_{p}=p_{p 0}$ in the $\mathrm{p}$-side material (the subscript $p$ denoting that it concerns the $\mathrm{p}$-side), $p_{p 0}$ being the equilibrium majority hole concentration, which is equal to the intrinsic hole concentration plus the doping one. In case of doping, the intrinsic carrier concentration is often to be neglected. For the n-side material, we can in analogy say that $n_{n} \gg p_{n}$ and $n_{n}=n_{n 0}$. Note that in equilibrium the product of the majority and minority carrier concentrations is a constant, being mathematically expressed by the so-called Mass Action Law

$p_{i 0} n_{i 0}=n_{i e}^{2}$

with subscript $i$ being either $p$ or $n$, depending whether it concerns the $\mathrm{p}$ - or the n-side, and $n_{i e}$ is the equilibrium carrier concentration. Therefore, at equilibrium, carrier concentrations are given by

$p_{p 0}=N_{A} \& n_{p 0}=n_{i e, p}^{2} / N_{A} \quad$ (where $\left.\left.n_{i e, p} \equiv n_{i e}\right|_{E_{g} \equiv E_{g p}}\right)$

for the majority and minority carriers in the p-side, respectively, and

$n_{n 0}=N_{D} \& p_{n 0}=n_{i e, n}^{2} / N_{D} \quad$ (where $\left.n_{i e, n} \equiv n_{i e}\right|_{E_{g} \equiv E_{g n}}$ )

for the majority and minority carriers in the n-side, respectively. Due to carrier doping, we also assume that $n_{n} \gg n_{i e}$ and $n_{p} \gg n_{i e}$. Furthermore, we assume that the lifetimes and surface recombination velocities do not vary dramatically in the $\mathrm{p}$ - and $\mathrm{n}$-side materials, such that $\tau n_{p} \gg \tau n_{n}$ in the p-side material and $\tau n_{n} \gg \tau n_{p}$ in the n-side material. Using Eqs. (59)-(62), the recombination rates for the minority carrier concentrations $\left(n_{p}-n_{p 0}\right)$ on the p-side and $\left(p_{n}-p_{n 0}\right)$ on the $\mathrm{n}$-side, respectively, become

$R_{n}=\left(n_{p}-n_{p 0}\right)\left(\frac{1}{\tau_{n}}+\frac{1}{\tau_{n s}}+\frac{1}{\tau_{n A}}\right)=\left(n_{p}-n_{p 0}\right) C_{R n}$

$R_{p}=\left(p_{n}-p_{n 0}\right)\left(\frac{1}{\tau_{p}}+\frac{1}{\tau_{p s}}+\frac{1}{\tau_{p A}}\right)=\left(p_{n}-p_{n 0}\right) C_{R p}$

$\tau_{n A} \equiv \frac{1}{B_{p} N_{A}^{2}}$

$\tau_{p A} \equiv \frac{1}{B_{n} N_{D}^{2}}$ 


$$
\begin{aligned}
& \tau_{n s}=\frac{W_{p}}{S_{r n}}+\frac{4}{D_{n}}\left(\frac{W_{p}}{\pi}\right)^{2} \\
& \tau_{p s}=\frac{W_{n}}{S_{r p}}+\frac{4}{D_{p}}\left(\frac{W_{n}}{\pi}\right)^{2}
\end{aligned}
$$

Here $1 / C_{R n}$ and $1 / C_{R p}$ are the effective recombination lifetimes of the minority electrons and holes, respectively. Very important here is to note that the electron and hole Auger lifetimes are obtained under the assumption that the dopant concentration is much higher than the minority ones [29]. If not, the Auger lifetimes should not be calculated as in (91a) or (91b) but can be obtained elsewhere, e.g. in [30, 31] for silicon, for instance. The expression for the surface recombination lifetime is given in [32], assuming identical surfaces and splitting the expression for the $\mathrm{p}$ - and n-sides. The set of equations to solve are now (noting that $\frac{d^{2} n_{p}}{d z^{2}}=\frac{d^{2}\left(n_{p}-n_{p 0}\right)}{d z^{2}}$ and $\left.\frac{d^{2} p_{n}}{d z^{2}}=\frac{d^{2}\left(p_{n}-p_{n 0}\right)}{d z^{2}}\right)$

$k_{B} T \frac{\mu_{n}}{e_{C}} \frac{d^{2}\left(n_{p}-n_{p 0}\right)}{d z^{2}}-C_{R n}\left(n_{p}-n_{p 0}\right)+C_{G p}=0$

$k_{B} T \frac{\mu_{p}}{e_{C}} \frac{d^{2}\left(p_{n}-p_{n 0}\right)}{d z^{2}}-C_{R p}\left(p_{n}-p_{n 0}\right)+C_{G n}=0$

for the minority carrier concentrations $\left(n_{p}-n_{p 0}\right)$ on the $\mathrm{p}$-side of the quasi-neutral region and $\left(p_{n}-p_{n 0}\right)$ minority carriers on the n-side of the quasi-neutral region, respectively. Let us furthermore define the characteristic diffusion lengths $L_{n} \equiv \sqrt{\frac{k_{B} T \mu_{n}}{C_{R n} e_{C}}}$ and $L_{p} \equiv \sqrt{\frac{k_{B} T \mu_{p}}{C_{R p} e_{C}}}$ and the characteristic diffusion coefficients $D_{n} \equiv \frac{k_{B} T \mu_{n}}{e_{C}}$ and $D_{p} \equiv \frac{k_{B} T \mu_{p}}{e_{C}}$ of the electrons (in the p-side) and the holes (in the n-side), respectively. We recall that $z=-z_{p}$ and $z=z_{n}$ at the edge of, respectively, the $\mathrm{p}$ - and n-side of the depletion region and $z \rightarrow-\left(W_{p}+z_{p}\right)$ and $z \rightarrow\left(W_{n}+z_{n}\right)$ for the surface of the device for the p- and n-sides, respectively, where $W_{p}$ and $W_{n}$ are the thickness of the $\mathrm{p}$-side and n-side quasi-neutral regions, respectively. On the $\mathrm{p}$-side we have the following boundary conditions:

$$
\begin{array}{ll}
D_{n} \frac{d\left(n_{p}-n_{p 0}\right)}{d z}=S_{r n}\left(n_{p}-n_{p 0}\right) & \text { at } z \rightarrow-\left(W_{p}+z_{p}\right) \\
\left(n_{p}-n_{p 0}\right)=n_{p 0} e^{\frac{e}{e V_{0}}}-n_{p 0} & \text { at } z=-z_{p}
\end{array}
$$

On the n-side we have the following boundary conditions:

$$
\begin{array}{ll}
-D_{p} \frac{d\left(p_{n}-p_{n 0}\right)}{d z}=S_{r p}\left(p_{n}-p_{n 0}\right) & \text { at } z \rightarrow\left(W_{n}+z_{n}\right) \\
\left(p_{n}-p_{n 0}\right)=p_{n 0} e^{\frac{e_{C} V_{0}}{k_{B} T}}-p_{n 0} & \text { at } z=z_{n}
\end{array}
$$

In (95) and (97), $V_{0}$ is the applied voltage or in the case of an illuminated solar cell the opencircuit voltage, here denoted by $V_{0} \equiv \Delta V$. Also $S_{r n}$ and $S_{r p}$ are the minority electron and hole surface recombination velocities, respectively. We find the following minority carrier densities in the p-side and the n-side, respectively 


$$
\begin{gathered}
n_{p}[z]=n_{p 0}+\frac{\left(e^{\frac{e_{C} \Delta V}{k_{B} T}}-1\right) n_{p 0} D_{n}\left(D_{n}-L_{n} S_{r n}+\left(D_{n}+L_{n} S_{r n}\right) e^{2 \frac{W_{p}+z_{p}+z}{L_{n}}}\right)}{e^{\frac{z p+z}{L_{n}}} D_{n}\left(D_{n}-L_{n} S_{r n}+\left(D_{n}+L_{n} S_{r n}\right) e^{\frac{2 W_{p}}{L_{n}}}\right)}- \\
\frac{C_{G p}\left(e^{\frac{z p+z}{L_{n}}}-1\right) L_{n}^{2}\left(D_{n}\left(e^{\frac{2 W_{p}+z_{p}+z}{L_{n}}}-1\right)+\left(e^{\frac{W_{p}}{L_{n}}}-1\right)\left(e^{\frac{W_{p}+z p+z}{L_{n}}}-1\right) L_{n} S_{r n}\right)}{e^{\frac{z}{L_{p}+z}} D_{n}\left(D_{n}-L_{n} S_{r n}+\left(D_{n}+L_{n} S_{r n}\right) e^{\frac{2 W_{p}}{L_{n}}}\right)} \\
p_{n}[z]=p_{n 0}+\frac{\left(e^{\frac{e_{C} \Delta V}{k_{B} T}}-1\right) p_{n 0} D_{p}\left(\left(D_{p}-L_{p} S_{r p}\right) e^{\frac{2 z}{L_{p}}}+\left(D_{p}+L_{p} S_{r p}\right) e^{2 \frac{W_{n}+z_{n}}{L_{p}}}\right)}{e^{\frac{z_{n}+z}{L_{p}}} D_{p}\left(D_{p}-L_{p} S_{r p}+\left(D_{p}+L_{p} S_{r p}\right) e^{\frac{2 W_{n}}{L_{p}}}\right)} \\
\frac{C_{G n}\left(e^{\frac{z}{L_{p}}}-e^{\frac{z_{n}}{L_{p}}}\right) L_{p}^{2}\left(D_{p}\left(e^{\frac{z}{L_{p}}}-e^{\frac{2 W_{n}+z_{n}}{L_{p}}}\right)+\left(e^{\frac{W_{n}}{L_{p}}}-1\right)\left(e^{\frac{z}{L_{p}}}-e^{\frac{W_{n}+z_{n}}{L_{p}}}\right) L_{p} S_{r p}\right)}{e^{\frac{z_{n}+z}{L_{p}}} D_{p}\left(D_{p}-L_{p} S_{r p}+\left(D_{p}+L_{p} S_{r p}\right) e^{\frac{2 W_{n}}{L_{p}}}\right)}
\end{gathered}
$$

The majority carrier currents are equal to zero in each of the respective quasi-neutral regions. The minority carrier currents can be calculated using the one-dimensional variants of equations (80)-(81) giving for the $\mathrm{p}$ - and $\mathrm{n}$-side, respectively,

$$
\begin{gathered}
I_{n}=e_{C} \frac{\left(e^{\frac{e_{C} \Delta V}{k_{B} T}}-1\right) n_{p 0} D_{n}\left(-D_{n}+L_{n} S_{r n}+\left(D_{n}+L_{n} S_{r n}\right) e^{2 \frac{W_{p}+z_{p}+z}{L_{n}}}\right)}{e^{\frac{z p+Z}{L_{n}}} L_{n}\left(D_{n}-L_{n} S_{r n}+\left(D_{n}+L_{n} S_{r n}\right) e^{\frac{2 W_{p}}{L_{n}}}\right)}- \\
\frac{C_{G p}\left(e^{\frac{z_{p}+z}{L_{n}}}-1\right) L_{n}^{2}\left(D_{n}\left(e^{2 \frac{W p+z_{p}+z}{L_{n}}}-1\right)+\left(e^{\frac{W_{p}}{L_{n}}}-1\right)\left(e^{\frac{W_{p}+2 z_{p}+2 z}{L_{n}}}-1\right) L_{n} S_{r n}\right)}{e^{\frac{z_{p}+z}{L_{n}}} L_{n}\left(D_{n}-L_{n} S_{r n}+\left(D_{n}+L_{n} S_{r n}\right) e^{\frac{2 W_{p}}{L_{n}}}\right)}
\end{gathered}
$$

$$
\begin{aligned}
& I_{P}= \\
& e_{C} \frac{2 e^{\frac{W_{n}}{L_{p}}}\left(C_{G n} L_{p}^{3} S_{r p} \operatorname{Cosh}\left(\frac{z-z_{n}}{L_{p}}\right)-\left(C_{G n} L_{p}^{2}-\left(e^{\frac{e_{C} \Delta V}{k_{B} T}}-1\right) p_{n 0} D_{p}\right)\left(L_{p} S_{r p} \operatorname{Cosh}\left(\frac{W_{n}-z+z_{n}}{L_{p}}\right)+D_{p} \operatorname{Sinh}\left(\frac{W_{n}-z+z_{n}}{L_{p}}\right)\right)\right)}{L_{p}\left(D_{p}-L_{p} S_{r p}+\left(D_{p}+L_{p} S_{r p}\right) e^{\frac{2 W_{n}}{L_{p}}}\right)}
\end{aligned}
$$

Having determined the minority current densities and the depletion region properties, we have enough information to proceed with the photovoltaic efficiency.

\subsection{Photovoltaic efficiency}

We make the assumption that the total current through the solar cell is constant:

$\frac{d I}{d z}=\frac{d I_{n}}{d z}+\frac{d I_{p}}{d z}=-e_{C}\left(G_{n}-R_{n}\right)+e_{C}\left(G_{p}-R_{p}\right)=0$

This is assumption can be made by stating that each electron generates a hole and each recombining electron uses one up: $G_{n}=G_{p}$ and $R_{n}=R_{p}$. This means that the total number of 
electrons and holes do not change in a semiconductor and therefore the total current also does not change. This also suggests that if one finds the total current anywhere in the device it will be the same everywhere in it. Conveniently, we can choose either sides of the depletion region. We choose to calculate the total current at the n-side, i.e. $z=z_{n}$. As stated earlier, both current densities across the depletion region (the edges for mathematical purposes included) are constant if it were not for the generation in the depletion region. So, the electron current density at the n-side of the depletion region $\left(z=z_{n}\right)$ equals the minority electron current density at the p-side of the depletion region $\left(z=-z_{p}\right)$ plus the one calculated due to generation in the depletion region (whether it be positive or negative): $I_{n}\left(z=z_{n}\right)=I_{n}\left(z=-z_{p}\right)+\Delta I_{n, d}$. The total current density can then be found by simply adding the hole current density at the n-side $I_{P}\left(z=z_{n}\right)$. Therefore, we can find the total current to be $I_{T}=I_{P}\left(z=z_{n}\right)+I_{n}\left(z=-z_{p}\right)+$ $\Delta I_{n, d}$. Recalling that $p_{n 0}=n_{i e}^{2} / N_{D}$ and $n_{p 0}=n_{i e}^{2} / N_{A}$, we find finally

$$
\begin{gathered}
I_{T}=\left(e^{\frac{e_{C} \Delta V}{k_{B} T}}-1\right)\left(e_{C} \frac{D_{p}}{L_{p}} \frac{n_{i e, n}^{2}}{N_{D}} \frac{\frac{D_{p}}{L_{p} S_{r p}}+\operatorname{Coth}\left(\frac{W_{n}}{L_{p}}\right)}{1+\frac{D_{p}}{L_{p} S_{r p}} \operatorname{Coth}\left(\frac{W_{n}}{L_{p}}\right)}+e_{C} \frac{D_{n}}{L_{n}} \frac{n_{i e, p}^{2}}{N_{A}} \frac{\frac{D_{n}}{L_{n} S_{r n}}+\operatorname{Coth}\left(\frac{W_{p}}{L_{n}}\right)}{1+\frac{D_{n}}{L_{n} S_{r n}} \operatorname{Coth}\left(\frac{W_{p}}{L_{n}}\right)}\right)-e_{C}\left(C_{G p} Z_{p}+\right. \\
\left.C_{G n} Z_{n}+C_{G n} L_{p} \frac{\frac{D_{p}}{L_{p} S_{r p}}+\operatorname{Tanh}\left(\frac{W_{n}}{2 L_{p}}\right)}{1+\frac{D_{p}}{L_{p} S_{r p}} \operatorname{Coth}\left(\frac{W_{n}}{L_{p}}\right)}+C_{G p} L_{n} \frac{\frac{D_{n}}{L_{n} S_{r n}}+\operatorname{Tanh}\left(\frac{W_{p}}{2 L_{n}}\right)}{1+\frac{D_{n}}{L_{n} S_{r n}} \operatorname{Coth}\left(\frac{W_{p}}{L_{n}}\right)}\right)
\end{gathered}
$$

We can typically define

$$
\begin{aligned}
& I_{0} \equiv e_{C} \frac{D_{p}}{L_{p}} \frac{n_{i e, n}^{2}}{N_{D}} \frac{\frac{D_{p}}{L_{p} S_{r p}}+\operatorname{Coth}\left(\frac{W_{n}}{L_{p}}\right)}{1+\frac{D_{p}}{L_{p} S_{r p}} \operatorname{Coth}\left(\frac{W_{n}}{L_{p}}\right)}+e_{C} \frac{D_{n}}{L_{n}} \frac{n_{i e, p}^{2}}{N_{A}} \frac{\frac{D_{n}}{L_{n} S_{r n}}+\operatorname{Coth}\left(\frac{W_{p}}{L_{n}}\right)}{1+\frac{D_{n}}{L_{n} S_{r n}} \operatorname{Coth}\left(\frac{W p}{L_{n}}\right)}, \\
& I_{S C} \equiv e_{C}\left(C_{G p} Z_{p}+C_{G n} Z_{n}+C_{G n} L_{p} \frac{\frac{D_{p}}{L_{p} S_{r p}}+\operatorname{Tanh}\left(\frac{W_{n}}{2 L_{p}}\right)}{1+\frac{D_{p}}{L_{p} S_{r p}} \operatorname{Coth}\left(\frac{W_{n}}{L_{p}}\right)}+C_{G p} L_{n} \frac{\frac{D_{n}}{L_{n} S_{r n}}+\operatorname{Tanh}\left(\frac{W_{p}}{2 L_{n}}\right)}{1+\frac{D_{n}}{L_{n} S_{r n}} \operatorname{Coth}\left(\frac{W_{p}}{L_{n}}\right)}\right)
\end{aligned}
$$

standing for the dark saturation current (without solar illumination, corresponding to the diode current) and the short-circuit current, respectively. It should be noted that the signs are rather based on conventions. When speaking about solar cells, expression (103) is often inversed in sign. Making the remark that in the case of solar cells, the short-circuit current is also called the light-generated current, i.e. $I_{L} \equiv I_{S C}$, we find the final expression for the total current

$I_{T}=I_{L}-I_{0}\left(e^{\frac{e_{C} \Delta V}{k_{B} T}}-1\right)$

It can be seen here that the total current density, for a specific set of $\mathrm{p}$ - and n-materials with a specific thickness depends on both the built-in and the induced voltage difference, $V_{D}$ and $\Delta V$. The first is material dependent and the second depends on the operating conditions. Therefore, $I_{T}=I_{T}(\Delta V)$. The photovoltaic efficiency $\eta_{p v}$ is then defined by the ratio of electric power $P_{p v}$ and the input solar power $P_{S}$

$$
\eta_{p v}=\frac{P_{p v}}{P_{S}}=\frac{\Delta V I_{T}(\Delta V)}{P_{S}}
$$

It is well known that an increasing $\Delta V$ decreases $I_{T}$ and can therefore expect a certain maximum in $\eta_{p v}$. Therefore, we can define a maximum photovoltaic efficiency as 
$\eta_{p v, \max }=\frac{\max \left(\Delta V I_{T}(\Delta V)\right)}{P_{S}}$

The optimal (subscript $o p t$ ) electric potential $\Delta V_{o p t}$ is the one for with Eq. (108) holds, so that the corresponding optimal current density will be

$I_{T, o p t}=\frac{\max \left(\Delta V I_{T}(\Delta V)\right)}{\Delta V_{\text {opt }}}$

We can then also define

$\eta_{p v, \max }=\frac{\Delta V_{o p t} I_{T, o p t}}{P_{S}}$

The maximum total efficiency of the photothermoelectric efficiency $\eta_{p v t e}$ is then given by

$\eta_{p v t e, \max }=\left(1-\eta_{p v, \max }\right) \eta_{t e, \max }+\eta_{p v, \max }$

We have developed models for both the thermoelectric and photovoltaic mechanism. Having said, that it is aimed to increase the photovoltaic efficiency by a cooled thermoelectric device, it is necessary to perform an analysis on the heat management of the entire cooled photovoltaicthermoelectric hybrid system in the next Section.

\section{Analysis of the heat management of the cooled hybrid system}

Before we can analyze the performance of the hybrid system, an analysis on the heat management should be performed. Let us recall for clarity that wherever an entity (for instance, the temperature) is used in both the photovoltaic and thermoelectric model, it will be distinguished by subscripts: the subscript te denotes the thermoelectric device and $p v$ the photovoltaic one. We can divide the heat flows in three parts. The first part is the net heat flow at the illuminated photovoltaic surface. The second part is the heat transfer from the photovoltaic device to the thermoelectric element. The third part is the heat removal from the thermoelectric element by the cooling device (which is powered by the photovoltaic device).

\subsection{Heat generation in the photovoltaic device}

The heat generation rate $H$ is given by the sum of the energy transferred to the lattice by lattice thermalization, $H_{h p}$, the energy of photons whose energy is lower than the band-gap energy, $H_{l p}$ and the joule heat, $H_{j}$ :

$H=H_{h p}+H_{l p}+H_{j}$

with

$H_{h p}=G \int_{k_{\min }}^{h c / E_{g}}\left(E_{\text {photon }}-E_{g}\right) \mathrm{d} k$

$H_{l p}=G \int_{h c / E_{g}}^{k_{\max }} E_{\text {photon }} \mathrm{d} k$

$H_{j}=-\nabla V \cdot\left(\boldsymbol{I}_{p}+\boldsymbol{I}_{n}\right)+E_{g} R$

where $G$ is given by (84) and (85) for the $\mathrm{n}$ - and p-side, respectively. The total heat generation rate is then expressed by 
$H=C_{H}+\frac{\Delta V_{o p t}}{\delta}\left(I_{p, o p t}+I_{n, o p t}\right)$

where the latter term is the joule heating, where we assume a linear relation for the voltage gradient with $\delta=\delta_{n}+\delta_{p}$ the total photovoltaic device thickness. The first term in (114) is given by

$$
\begin{aligned}
& C_{H}=\frac{\delta_{p}}{\delta_{p}+\delta_{n}} C_{H p}+\frac{\delta_{n}}{\delta_{p}+\delta_{n}} C_{H n} \\
& C_{H p}=C_{G p}\left(E_{g p} k_{\text {min }}-h c+\frac{h c}{2 \pi} \ln \left(\frac{k_{\max }}{k_{\min }}\right)\right)+E_{g p} R_{n, b u l k} \\
& C_{H n}=C_{G n}\left(E_{g n} k_{\text {min }}-h c+\frac{h c}{2 \pi} \ln \left(\frac{k_{\max }}{k_{\min }}\right)\right)+E_{g n} R_{p, b u l k}
\end{aligned}
$$

In (115a), we have taken into account the fact that a thicker layer will generate more heat. Moreover, we distinguish between the heat generated in the $\mathrm{p}$ - and n-sides of the photovoltaic device, the differences being given by the respective thicknesses $\delta_{p}$ and $\delta_{n}$, the respective band gap energies $E_{g p}$ and $E_{g n}$, the respective generation rates $C_{g p}$ and $C_{g n}$, and the respective recombination rates $R_{n, b u l k}$ and $R_{p, b u l k}$. Note that the subscripts of the recombination rates are different now. On one hand, it concerns the recombination of electrons or holes (subscript $n$ or $p$ for the recombination rate) in the $\mathrm{p}$ - or $\mathrm{n}$-side (subscript $p$ or $n$ for the heat generation). On the other hand, we only consider the bulk recombination rate as far as it concerns the heat generation. For this, we take Eqs. (89) and (90), where we fill in Eqs. (98) and (99) and take then the average value of the carrier concentrations over the respective quasi-neutral region. In doing so, we do not consider the contribution of the surface recombination in $C_{R n}$ (see Eq. (89)) en $C_{R p}$ (see Eq. (90)), respectively. This results finally into

$$
\begin{aligned}
& R_{n, b u l k} \approx \frac{1}{W_{p}} \int_{-W_{p}-z_{p}}^{-z_{p}}\left(n_{p}-n_{p 0}\right) C_{R p} d z=\left(\frac{1}{\tau_{n}}+\frac{1}{\tau_{n A}}\right) \times \\
& \frac{\left(e^{\frac{e_{C} \Delta V}{k_{B} T}}-1\right) n_{p 0} D_{n}\left(L_{n} S_{r n}\left(\operatorname{Cosh}\left(\frac{W_{p}}{L_{n}}\right)-1\right)+D_{n} \operatorname{Sinh}\left(\frac{W_{p}}{L_{n}}\right)\right)}{\frac{D_{n} W_{p}}{2 L_{n}} e^{-\frac{W_{p}}{L_{n}}}\left(D_{n}-L_{n} S_{r n}+\left(D_{n}+L_{n} S_{r n}\right) e^{\frac{2 W_{p}}{L_{n}}}\right)}+\left(\frac{1}{\tau_{n}}+\frac{1}{\tau_{n A}}\right) \times \\
& \frac{C_{G p} L_{n}\left(\left(D_{n} W_{p}-2 L_{n}^{2} S_{r n}\right) \cosh \left(\frac{W_{p}}{L_{n}}\right)+L_{n}\left(2 L_{n} S_{r n}+\left(W_{p} S_{r n}-D_{n}\right) \sinh \left(\frac{W_{p}}{L_{n}}\right)\right)\right)}{\frac{D_{n} W_{p}}{2 L_{n}} e^{-\frac{W_{p}}{L_{n}}}\left(D_{n}-L_{n} S_{r n}+\left(D_{n}+L_{n} S_{r n}\right) e^{\frac{2 W_{p}}{L_{n}}}\right)} \\
& R_{n, b u l k} \approx \frac{1}{W_{n}} \int_{z_{n}}^{W_{n}+z_{n}}\left(p_{n}-p_{n 0}\right) C_{R n} d z=\left(\frac{1}{\tau_{p}}+\frac{1}{\tau_{p A}}\right) \times \\
& \frac{\left(e^{\frac{e_{C} \Delta V}{k_{B} T}}-1\right) p_{n 0} D_{p}\left(L_{p} S_{r p}\left(\cosh \left(\frac{W_{n}}{L_{p}}\right)-1\right)+D_{p} \sinh \left(\frac{W_{n}}{L_{p}}\right)\right)}{\frac{D_{p} W_{n}}{2 L_{p}} e^{-\frac{W_{n}}{L_{p}}}\left(D_{p}-L_{p} S_{r p}+\left(D_{p}+L_{p} S_{r p}\right) e^{\frac{2 W_{n}}{L_{p}}}\right)}+\left(\frac{1}{\tau_{p}}+\frac{1}{\tau_{p A}}\right) \times \\
& \frac{C_{G n} L_{p}\left(\left(D_{p} W_{n}-2 L_{p}^{2} S_{r p}\right) \operatorname{Cosh}\left(\frac{W_{n}}{L_{p}}\right)+L_{p}\left(2 L_{p} S_{r p}+\left(W_{n} S_{r p}-D_{p}\right) \sinh \left(\frac{W_{n}}{L_{p}}\right)\right)\right)}{\frac{D_{p} W_{n}}{2 L_{p}} e^{-\frac{W_{n}}{L_{p}}\left(D_{p}-L_{p} S_{r p}+\left(D_{p}+L_{p} S_{r p}\right) e^{\frac{2 W_{n}}{L_{p}}}\right)}}
\end{aligned}
$$


where $\Delta V=\Delta V_{p v, o p t}$ corresponding to the one where Eq. (108) holds. The last term of (114) is the so-called Joule heating, obtained at optimal conditions, defined by (108)-(109).

\subsection{Temperature profiles}

The quasi-stationary energy balance equation in the photovoltaic device is given by

$\nabla \cdot\left(\lambda_{p v} \nabla T_{p v}\right)+H=0$

where $\lambda_{p v}$ is the thermal conductivity of the photovoltaic device. We define an overall thermal conductivity of the photovoltaic device:

$\lambda_{p v}=\frac{\delta_{n}+\delta_{p}}{\frac{\delta_{n}}{\lambda_{p v n}}+\frac{\delta_{p}}{\lambda_{p v p}}}$

where the subscripts $\lambda_{p v n}$ and $\lambda_{p v p}$ denote the thermal conductivities of the $\mathrm{n}$ - and $\mathrm{p}$-side materials of the photovoltaic device and $\delta_{n}$ and $\delta_{p}$ the n- and p-sides' total thicknesses, respectively. At the upper surface of the photovoltaic device, we assume that the only heat transfer mechanisms are solar radiation $P_{S}$ (increasing the surface temperature) and heat convection (decreasing the surface temperature). The boundary condition at the upper surface is then

$-\left.\lambda_{p v} \frac{\partial \mathrm{T}_{p v}}{\partial \mathrm{y}}\right|_{y=0}=P_{s}-h_{s}\left(\mathrm{~T}(y=0)-\mathrm{T}_{a m b}\right)$

with $y=0$ at the illuminated upper surface and $y=\delta$ at the dark side. We choose in this section to use a different symbol for the space coordinate just for the sake of clarity and convenience. In (120), $h_{s}$ stands for the convective heat transfer coefficient towards the ambient air with temperature $\mathrm{T}_{a m b}$. Since $h_{s}$ is variable due to external factors, we will take a typical mean value of $h_{s}=10 \mathrm{Wm}^{-2} \mathrm{~K}^{-1}$ [33]. We can see from (11a) and (11b) that under the assumptions of this work the heat flux in the thermoelectric device is considered to be constant. Assuming heat flux continuity through the photovoltaic-thermoelectric interface, we have the following boundary condition at $y=\delta$ :

$$
\begin{aligned}
& -\left.\lambda_{p v} \frac{\partial \mathrm{T}_{p v}}{\partial \mathrm{y}}\right|_{y=\delta}=q_{p v, o p t} \\
& q_{p v, o p t}=q_{t e, o p t}
\end{aligned}
$$

with $q_{t e, o p t}$ the optimal heat flux from the sum of (11a) and (11b), wherein the material properties are taken at $T_{r e f}$ and the optimal thermoelectric current density $I_{t e, o p t}$ is used from Eq. (16). Using (18), the total heat flux in the thermoelectric element can also be written as

$q_{t e, o p t}=\lambda_{t e, t o t} \frac{T_{t e, h}-T_{t e, c}}{L} \sqrt{1+Z T}$

Let us make some short reflections on (122). If the Peltier effect was neglected, Eq. (122) would reduce to the simple Fourier law. For bulk materials, $Z T$ is a positive constant. For nanocomposites, the case in this paper, $Z T$ depends on the nanoparticle radius and volume fraction via the size-dependent material properties. Recalling that the thermoelectric device 
consists of a p- and an n-leg, the total effective thermal conductivity is derived by means of the Matthiesen rule uniquely for the purposes of the present section:

$\lambda_{t e, t o t}=\left(\frac{1}{\lambda_{p h, p}+\lambda_{e, p}}+\frac{1}{\lambda_{p h, n}+\lambda_{e, n}}\right)^{-1}$

Having calculated the current through the photovoltaic device, we can through Eqs. (118) and (119)-(123) calculate the maximum generated heat. The n-side layer is often much smaller than the p-side layer, so that we can neglect the effect of the former's thickness on the temperature variation. We solve Eq. (118) at optimal conditions and find the following temperature profile in the photovoltaic device

$T_{p v, h}(z)=T_{a m b}+\frac{P_{s}-q_{t e, o p t}+H \delta}{h_{s}}-\frac{\left(H z+2 q_{t e, o p t}-2 H \delta\right) z}{2 \lambda_{p v}}$

The temperature at the photovoltaic-thermoelectric interface is thus

$T_{p v, h}=T(y=\delta)=T_{a m b}+\frac{P_{s}-q_{t e, o p t}+H \delta}{h_{s}}+\frac{H \delta^{2}-2 \delta q_{t e, o p t}}{2 \lambda_{p v}}$

\subsection{Operating temperatures}

In order to perform all the calculations concerning the photovoltaic device, we have seen in Sections 2 to 4 that there is a temperature dependence. It is not the purpose here to study this temperature dependence, but in order to have realistic values, we should evaluate the calculations for the photovoltaic device at a certain realistic operating temperature, valid wherever no high $\left(T_{h}\right)$ or low $\left(T_{c}\right)$ temperature is explicitly specified. We define this operating temperature $T \equiv T_{o p, p v}$ as an approximation of (125), valid for $P_{S} \gg q_{t e, o p t}$ and $P_{S} \gg H \delta$ :

$T_{o p, p v}=T_{a m b}+\frac{P_{s}}{h_{s}}$

Concerning the thermoelectric device, we assume that it operates under an average temperature between the photovoltaic one and the low cooling temperature:

$T_{o p, t e}=\frac{T_{o p, p v}+T_{e n v}}{2}$

As far as concerns material properties, we assume they are evaluated at a constant ambient temperature. We assume temperature continuity through the photovoltaic-thermoelectric interface, so that we define $T_{p v, h} \equiv T_{t e, h}$. Using a simple Newton's law of cooling we can state that at quasi-stationary conditions, the heat (assumed to be constant) through the thermoelectric device is equal to

$q_{t e, o p t}=h_{c}\left(T_{t e, c}-T_{e n v}\right)$

where $h_{c}$ is the convective heat transfer coefficient towards the cooling device (e.g. air fin, heat pipe) and $T_{e n v}$ the temperature far away from the contact surface (possibly, but not necessarily equal to $\left.T_{a m b}\right)$. The heat transfer coefficient towards the cooling air, $h_{c}$, can be found [7] by

$h_{c}=\frac{\lambda_{c} N u}{L_{c}}$ 
$N u=0.664 \operatorname{Re}^{1 / 2} \operatorname{Pr}^{1 / 3}$

$R e=\frac{\rho_{c} v_{c} L_{c}}{\mu_{c}}$

$\operatorname{Pr}=\frac{c_{p, c} \mu_{c}}{\lambda_{c}}$

where $N u, \operatorname{Re}, \operatorname{Pr}, \lambda_{c}, \rho_{c}, v_{c}, \mu_{c}, c_{p, c}$ and $L_{c}$ are the dimensionless Nusselt, Reynolds and Prandtl numbers, the thermal conductivity, density, velocity, dynamic viscosity, heat capacity of the cooling fluid and the characteristic length (typically the length of the hybrid system) at which the cooling takes place, respectively. The power flux $P_{c}$ needed for cooling depends on the flow mean velocity $v_{c}$ and the pressure drop $\Delta P$ to overcome:

$P_{c}=v_{c} \Delta P$

where the pressure drop is obtained via the standard Hagen-Poiseuille equation for laminar flow between two horizontal plates:

$\Delta P=\frac{12 \mu_{c} L_{c} v_{c}}{W_{c}^{2}}$

where $W_{c}$ is the depth of the cooling channel.

Rearranging Eqs. (122), (125) and (128) gives finally the expressions for $T_{t e, h}$ and $T_{t e, c}$ :

$$
\begin{aligned}
& T_{t e, h}=\frac{h_{c} L\left(h_{s} H \delta^{2}+2 \lambda_{p v}\left(P_{S}+h_{s} T_{a m b}+H \delta\right)\right)}{2 h_{c} h_{S} L \lambda_{p v}+2 \lambda_{t e, t o t} \sqrt{1+Z T}\left(h_{c} h_{S} \delta+\lambda_{p v}\left(h_{c}+h_{S}\right)\right)}+ \\
& \frac{\lambda_{t e, t o t} \sqrt{1+Z T}\left(h_{S} \delta\left(2 h_{c} T_{e n v}+H \delta\right)+2 \lambda_{p v}\left(P_{S}+h_{S} T_{a m b}+H \delta+h_{c} T_{e n v}\right)\right)}{2 h_{c} h_{S} L \lambda_{p v}+2 \lambda_{t e, t o t} \sqrt{1+Z T}\left(h_{c} h_{S} \delta+\lambda_{p v}\left(h_{c}+h_{S}\right)\right)} \\
& T_{t e, c}=\frac{h_{c} L T_{e n v}+T_{t e, h} \lambda_{t e, t o t} \sqrt{1+Z T}}{h_{c} L+\lambda_{t e, t o t} \sqrt{1+Z T}}
\end{aligned}
$$

It is interesting to note that the $N u$ number in Eq. (130) is linked to the $K n$ number via Eqs. (128), (129) and (136). If the cooling area would be a nano-channel, the correlation in Eq. (130) would not be valid and would also be dependent explicitly on the $K n$ number [34].

\subsection{Total efficiency of the hybrid system}

It is the idea to extract the power flux for the cooling device directly from the electricity produced from the photovoltaic device, which causes a loss of efficiency equal to

$\eta_{c}=\frac{P_{c}}{P_{S}}$

The net efficiency of the total hybrid system is then

$\eta_{\text {net }}=\eta_{p v, \max }+\left(1-\eta_{p v, \max }\right) \eta_{t e, \max }-\eta_{c}$

The net efficiency in this work is considered to be influenced by four parameters: the photovoltaic thickness $\delta$, the thermoelectric thickness $L$, the nanoparticle radius in the thermoelectric material $r_{p}$ and its volume fraction $\varphi$, and the cooling velocity $v_{c}$. The material and operating values are given and discussed in Section 6. 


\section{Material properties and operating conditions 6.1 Material properties for the thermoelectric materials}

The p-leg of the thermoelectric element is composed out of p-type Antimony Telluride ( $\mathrm{p}$ $\mathrm{Sb}_{2} \mathrm{Te}_{3}$ ) nanoparticles dispersed in a matrix of p-type Bismuth Telluride ( $\left.\mathrm{p}-\mathrm{Bi}_{2} \mathrm{Te}_{3}\right)$. The n-leg is composed out of n-type Bismuth Selenide $\left(\mathrm{n}-\mathrm{Bi}_{2} \mathrm{Se}_{3}\right)$ nanoparticles dispersed in a matrix of n-type Bismuth Telluride ( $\mathrm{n}-\mathrm{Bi}_{2} \mathrm{Te}_{3}$ ).

We start with $\mathrm{p}-\mathrm{Bi}_{2} \mathrm{Te}_{3}$. The electron $\left(C_{e}, v_{e}\right.$ and $\left.\Lambda_{e, b}\right)$ and phonon $\left(C_{p h}, v_{p h}\right.$ and $\left.\Lambda_{p h, b}\right)$ material properties are given by $[5,6]$ and the calculated thermal conductivities correspond with the ones in [35]. As for the Seebeck coefficient we use the value from [5]: $188 \mu \mathrm{V} / \mathrm{K}$. The electric conductivity can be found by (24) and (27), so that the electric conductivity is found to be $3.28 \times 10^{5} \Omega^{-1} \mathrm{~m}^{-1}$.

As for p-type Antimony Telluride ( $\left.\mathrm{Sb}_{2} \mathrm{Te}_{3}\right)$, the electric conductivity is $4.76 \times 10^{5} \Omega^{-1} \mathrm{~m}^{-1}$ [36]. Via (27), we can calculate the electron thermal conductivity to be $3.49 \mathrm{~W} / \mathrm{Km}$. Given that the total thermal conductivity is $4.2 \mathrm{~W} / \mathrm{Km}$ [37], we can deduce that the phonon thermal conductivity is $0.71 \mathrm{~W} / \mathrm{Km}$. The Seebeck coefficient and the phonon velocity are $79 \mu \mathrm{V} / \mathrm{K}$ and $2900 \mathrm{~m} / \mathrm{s}$, respectively [37]. The total specific heat capacity is $1.34 \mathrm{MJ} / \mathrm{m}^{3} \mathrm{~K}[38,39]$. For the phonon mean free path, it can be found that it is $3 \mathrm{~nm}$ [40]. We can then via (24) calculate that the phonon contribution of the specific heat is equal to $0.245 \mathrm{MJ} / \mathrm{m}^{3} \mathrm{~K}$. Assuming that the total specific heat is the sum of the phonon and electron contributions [5], the electron contribution of the specific heat is then equal to $1.09 \mathrm{MJ} / \mathrm{m}^{3} \mathrm{~K}$. The electron mean free path is $1 \mathrm{~nm}$ [41]. This makes via (24) the thermal electron velocity equaling $9562 \mathrm{~m} / \mathrm{s}$.

We assume that for the n-type $\mathrm{Bi}_{2} \mathrm{Te}_{3}$ at room temperature the phonon and electron mean free paths as well as the total specific heats remain the same as for the p-type materials. As for the n-type $\mathrm{Bi}_{2} \mathrm{Te}_{3}$, the total thermal conductivity is $1.27 \mathrm{~W} / \mathrm{Km}$, while the electric conductivity equals $0.96 \times 10^{5} \Omega^{-1} \mathrm{~m}^{-1}$ [42]. The latter results via (27) into an electron thermal conductivity of $0.70 \mathrm{~W} / \mathrm{Km}$ and according to the definition under Eq. (14) a phonon thermal conductivity of $0.57 \mathrm{~W} / \mathrm{Km}$. The phonon velocity is given to be $1750 \mathrm{~m} / \mathrm{s}$ [43]. This gives via (24) a phonon specific heat of $0.324 \mathrm{MJ} / \mathrm{m}^{3} \mathrm{~K}$ and, knowing that the total specific heat is $1.2 \mathrm{MJ} / \mathrm{m}^{3} \mathrm{~K}[5,6]$, thus an electron specific heat of $0.876 \mathrm{MJ} / \mathrm{m}^{3} \mathrm{~K}$. The thermal electron velocity is via (24) therefore $2634 \mathrm{~m} / \mathrm{s}$. The Seebeck coefficient is $-241 \mu \mathrm{V} / \mathrm{K}$ [3].

It remains to evaluate the properties of n-type Bismuth Selenide $\left(\mathrm{Bi}_{2} \mathrm{Se}_{3}\right)$. The electric conductivity is $2.4 \times 10^{5} \Omega^{-1} \mathrm{~m}^{-1}$ [3], which gives via (27) an electron thermal conductivity of $1.76 \mathrm{~W} / \mathrm{Km}$. Knowing from [3] that the phonon thermal conductivity is $0.66 \mathrm{~W} / \mathrm{Km}$, the total thermal conductivity is thus $2.42 \mathrm{~W} / \mathrm{Km}$. The phonon velocity is $2900 \mathrm{~m} / \mathrm{s}$ [44]. For the phonon specific heat, we have obtained the value from $[45,46]$ being $0.255 \mathrm{MJ} / \mathrm{m}^{3} \mathrm{~K}$. We can deduce via (24) that the phonon mean free path is $2.7 \mathrm{~nm}$. The total heat capacity being $1.33 \mathrm{MJ} / \mathrm{m}^{3} \mathrm{~K}$ [3], we can calculate the electron heat capacity to be $1.08 \mathrm{MJ} / \mathrm{m}^{3} \mathrm{~K}$. The Seebeck coefficient is $-50 \mu \mathrm{V} / \mathrm{K}$ [3]. The electron mean free path is said to be approximately $1 \mathrm{~nm}$ [47]. We can then via (24) finally calculate the electron velocity to be $4889 \mathrm{~m} / \mathrm{s}$.

A resume of these properties are given in Table 1, where we indicate whether it concerns the nanoparticles (NP) or the matrix (M).

Table 1: Electron and phonon material properties of $\mathrm{p}-\mathrm{Bi}_{2} \mathrm{Te}_{3}$ and $\mathrm{n}-\mathrm{Bi}_{2} \mathrm{Te}_{3}, \mathrm{p}-\mathrm{Sb}_{2} \mathrm{Te}_{3}$ and $\mathrm{n}-$ $\mathrm{Bi}_{2} \mathrm{Se}_{3}$ at $\mathrm{T}=300 \mathrm{~K}$.

\begin{tabular}{|cccccccccc|}
\hline Material & Role & \multicolumn{3}{c}{ Electron } & \multicolumn{3}{c}{ Phonon } & \multicolumn{2}{c|}{ Electrical } \\
& & $C_{e}$ & $v_{e}$ & $\Lambda_{e, b}$ & $C_{p h}$ & $v_{p h}$ & $\Lambda_{p h, b}$ & $\sigma_{e}$ & $S_{b}$ \\
& & {$\left[\mathrm{MJ} / \mathrm{m}^{3} \mathrm{~K}\right]$} & {$[\mathrm{km} / \mathrm{s}]$} & {$[\mathrm{nm}]$} & {$\left[\mathrm{MJ} / \mathrm{m}^{3} \mathrm{~K}\right]$} & {$[\mathrm{km} / \mathrm{s}]$} & {$[\mathrm{nm}]$} & {$\left[\mathrm{k} \Omega^{-1} \mathrm{~m}^{-1}\right]$} & {$[\mu \mathrm{V} / \mathrm{K}]$} \\
\hline
\end{tabular}




\begin{tabular}{|llllllllll|}
\hline $\mathrm{p}-\mathrm{Sb}_{2} \mathrm{Te}_{3}$ & $\mathrm{NP}$ & 1.09 & 9.65 & 1.0 & 0.245 & 2.9 & 3.0 & 476 & 79 \\
$\mathrm{p}-\mathrm{Bi}_{2} \mathrm{Te}_{3}$ & $\mathrm{M}$ & 1.01 & 7.83 & 0.91 & 0.19 & 8.43 & 3.0 & 328 & 188 \\
$\mathrm{n}-\mathrm{Bi}_{2} \mathrm{Se}_{3}$ & $\mathrm{NP}$ & 1.08 & 4.89 & 1.0 & 0.255 & 2.9 & 2.7 & 250 & -50 \\
$\mathrm{n}-\mathrm{Bi}_{2} \mathrm{Te}_{3}$ & $\mathrm{M}$ & 0.876 & 2.63 & 0.91 & 0.324 & 1.75 & 3.0 & 96 & -241 \\
\hline
\end{tabular}

\subsection{Material properties for the photovoltaic materials}

We consider in this work two types of photovoltaic materials. One is monocrystalline Silicon (c-Si) and thin-film polycrystalline Silicon (p-Si, not to be confused with the p-side of a solar cell, which is considered in both the Si materials). The electron and hole properties of c-Si and p-Si are shown in Table 2. Typical doping concentrations are taken from [48] and presented in Table 3. The hole Auger lifetime is extracted from Auger lifetime studies [29, 49] as $\tau_{p A}=$ $1 /\left(B_{n} N_{\text {dop }}^{2}\right)$ and $\tau_{n A}=1 /\left(B_{p} N_{d o p}^{2}\right)$, where $N_{\text {dop }}=N_{D}, N_{A}$ is the net dopant concentration in the $\mathrm{p}$ - and n-sides, respectively. This expression is only valid for net dopant concentrations $N_{\text {dop }}>5 * 10^{24} \mathrm{~m}^{-3}$, which is here only the case for the n-side of both the Si solar cells (see doping concentrations in Table 3). For the p-side, we extract the value from [30, 31] to be $\tau_{n A}=$ $8 * 10^{-5} \mathrm{~s}$. The Auger recombination coefficients $B_{p}$ and $B_{n}$ are taken from [29]. The effective densities of state are taken from [7]. All these mentioned values are taken to be the same for c$\mathrm{Si}$ and $\mathrm{p}-\mathrm{Si}$. The differences are in the SRH lifetimes, the electron and hole mobilities and the absorption coefficient. The SRH lifetimes $\tau_{p}$ and $\tau_{n}$ for $\mathrm{p}$-Si are obtained from the Scharfetter relation [28], which represents them as a function of the total dopant concentration:

$$
\tau_{n, p}=\frac{\tau_{\max , n, p}}{\left(\frac{N_{D}+N_{A}}{N_{n, p, r e f}}\right)^{\gamma}}
$$

The parameters for this relation are given in [28]. For $\tau_{\max , n}=10^{-5} \mathrm{~s}, \tau_{\max , p}=3 * 10^{-6} \mathrm{~s}$, $N_{n, p, r e f}=10^{22} \mathrm{~m}^{-3}$ and $\gamma=1$, we obtain the SRH lifetimes given in Table 2. The SRH lifetimes for c-Si are extracted from [50]. The mobility's for $\mathrm{p}-\mathrm{Si}$ depend on the doping concentrations and this can well be approximated by $[51,52]$

$$
\begin{gathered}
\mu_{n}=92 * 10^{-4}+\frac{1268 * 10^{-4}}{1+\left(\frac{N_{D}+N_{A}}{1.3 * 10^{23}}\right)^{0.91}} \\
\mu_{p}=54.3 * 10^{-4}+\frac{406.9 * 10^{-4}}{1+\left(\frac{N_{D}+N_{A}}{2.35 * 10^{23}}\right)^{0.88}}
\end{gathered}
$$

The mobility's for c-Si are taken from [7]. The absorption coefficient for p-Si is much higher than for c-Si [53]. For the absorption coefficient, we have taken a value averaged over the whole considered wavelength spectrum following the example of a correlation given in [54].

Table 2: Electron and hole material properties of c-Si and p-Si thin film at $\mathrm{T}=300 \mathrm{~K}$.

\begin{tabular}{|c|c|c|c|}
\hline $\begin{array}{c}\text { General material } \\
\text { property }\end{array}$ & Hole & $\begin{array}{c}\text { General material } \\
\text { property }\end{array}$ & Electron \\
\hline$\tau_{p A}[\mathrm{~s}]$ & $3.6 \times 10^{-10}$ & $\tau_{n A}[\mathrm{~s}]$ & $8.5 \times 10^{-5}$ \\
\hline$B_{p}\left[\mathrm{~m}^{6} / \mathrm{s}\right]$ & $9.9 \times 10^{-44}$ & $B_{n}\left[\mathrm{~m}^{6} / \mathrm{s}\right]$ & $2.8 \times 10^{-43}$ \\
\hline$N_{V}\left[\mathrm{~m}^{-3}\right]$ & $1.04 \times 10^{25}$ & $N_{C}\left[\mathrm{~m}^{-3}\right]$ & $2.8 \times 10^{25}$ \\
\hline
\end{tabular}




\begin{tabular}{|c|c|c|c|c|c|}
\hline $\begin{array}{c}\text { Specific material } \\
\text { property }\end{array}$ & $\mathrm{c}-\mathrm{Si}$ & $\mathrm{p}-\mathrm{Si}$ & $\begin{array}{c}\text { Specific material } \\
\text { property }\end{array}$ & $\mathrm{c}-\mathrm{Si}$ & $\mathrm{p}-\mathrm{Si}$ \\
\hline$\mu_{p}\left[\mathrm{~m}^{2} / \mathrm{Vs}\right]$ & $5 \times 10^{-2}$ & $56.3 \times 10^{-4}$ & $\mu_{n}\left[\mathrm{~m}^{2} / \mathrm{Vs}\right]$ & $1 \times 10^{-1}$ & $95.0 \times 10^{-4}$ \\
\hline$\tau_{p}[\mathrm{~s}]$ & $1 \times 10^{-6}$ & $3 \times 10^{-10}$ & $\tau_{n}[\mathrm{~s}]$ & $1 \times 10^{-6}$ & $1 \times 10^{-9}$ \\
\hline
\end{tabular}

Other photon and carrier information is given in Table 3. Note that the permittivity $\varepsilon$ is given as the relative one $\varepsilon_{r}$ (in Table 3 ) multiplied by the vacuum one $\varepsilon_{0}$, i.e. $\varepsilon=\varepsilon_{r} \varepsilon_{0}$. The thermal conductivity for monocrystalline silicon and polycrystalline silicon are taken from [55] and [56], respectively.

Table 3: Other material properties of $\mathrm{c}-\mathrm{Si}$ and $\mathrm{p}-\mathrm{Si}$ thin film at $\mathrm{T}=300 \mathrm{~K}$.

\begin{tabular}{|c|c|c|}
\hline Material property & $\mathrm{c}-\mathrm{Si}$ & $\mathrm{p}-\mathrm{Si}$ \\
\hline$E_{g}[\mathrm{eV}]$ & 1.12 & 1.12 \\
\hline$\varepsilon_{r}[-]$ & 11.8 & 11.7 \\
\hline$N_{A}\left[\mathrm{~m}^{-3}\right]$ & $1 \times 10^{22}$ & $1 \times 10^{22}$ \\
\hline$N_{D}\left[\mathrm{~m}^{-3}\right]$ & $1 \times 10^{26}$ & $1 \times 10^{26}$ \\
\hline$\alpha\left[\mathrm{m}^{-1}\right]$ & $4 \times 10^{3}$ & $2.5 \times 10^{5}$ \\
\hline$\lambda_{p v}[\mathrm{~W} / \mathrm{Km}]$ & 148 & 20 \\
\hline
\end{tabular}

Finally, it should be noted that although some properties of the considered materials in this paper have the same values in the $\mathrm{n}$ - and $\mathrm{p}$-sides, they are formally different in the photovoltaic model in this paper. This is done so that the model remains generally applicable to other photovoltaic materials, where the $\mathrm{n}$ - and $\mathrm{p}$-sides are made out of distinctly different materials.

\subsection{Other operating characteristics and general physical properties}

Table 4 presents the characteristics of the cooler and Table 5 presents some general physical constants used throughout this paper.

Table 4: Cooler characteristics [7]

\begin{tabular}{|l|l|}
\hline Property & Value \\
\hline$h_{S}\left[\mathrm{~W} / \mathrm{Km}^{2}\right]$ & 10 \\
\hline$P_{S}\left[\mathrm{~W} / \mathrm{m}^{2}\right]$ & 1000 \\
\hline$\lambda_{c}[\mathrm{~W} / \mathrm{Km}]$ & 0.0242 \\
\hline$L_{c}[\mathrm{~m}]$ & 0.025 \\
\hline$\rho_{c}\left[\mathrm{~kg} / \mathrm{m}^{3}\right]$ & 1.2 \\
\hline$\mu_{c}[\mathrm{~Pa} \mathrm{~s}]$ & $1.84 \times 10^{-5}$ \\
\hline$c_{p, c}[\mathrm{~J} / \mathrm{kgK}]$ & 1007 \\
\hline$W_{c}[\mathrm{~m}]$ & 0.025 \\
\hline
\end{tabular}

Table 5: Physical constants at $\mathrm{T}=300 \mathrm{~K}$.

\begin{tabular}{|l|llllll|}
\hline Physical & $k_{B}$ & $h$ & $e_{c}$ & $L$ & $\mathrm{c}$ & $\varepsilon_{0}$ \\
constant & {$\left[10^{-23} \mathrm{~J} / \mathrm{K}\right]$} & {$\left[10^{-34} \mathrm{Js}\right]$} & {$\left[10^{-19} \mathrm{C}\right]$} & {$\left[10^{-8} \mathrm{~W} \Omega / \mathrm{K}^{2}\right]$} & {$\left[10^{8} \mathrm{~m} / \mathrm{s}\right]$} & {$\left[10^{-12} \mathrm{C} / \mathrm{Vm}\right]$} \\
Value & 1.38 & 6.626 & 1.602 & 2.44 & 3.0 & 8.854 \\
\hline
\end{tabular}

Setting $T_{a m b}=T_{e n v}=300 \mathrm{~K}$, we can deduce from Eqs. (126) and (127), the operating temperatures $T_{o p, p v}=400 \mathrm{~K}$ and $T_{o p, t e}=350 \mathrm{~K}$. 


\section{Results}

\subsection{Optimal thickness of the photovoltaic device}

First of all, we investigate the influence of the thickness of the photovoltaic device and the surface recombination velocity on the electric power. Note that the photovoltaic efficiency is this electric power output divided by the solar power input $P_{S}=1000 \mathrm{~W} / \mathrm{m}^{2}$. As for the surface recombination velocity, we assume that it is equal in n- and p-sides, although it is typically somewhat smaller in the n-side [57]. So, we take $S_{r p}=S_{r n}=S_{r}$. Fig. 2 shows the photovoltaic power output as a function of the p-side thickness for four n-side thicknesses for c-Si, i.e. $\delta_{n}=$ $50 \mathrm{~nm}, \delta_{n}=250 \mathrm{~nm}, \delta_{n}=2 \mu \mathrm{m}$ and $\delta_{n}=100 \mu \mathrm{m}$. The same for p-Si is shown in Fig. 3. The $\delta_{n}$ value of $50 \mathrm{~nm}$ appears to be the smallest manufacturable n-side thickness for the doping concentration used in this work [48]. The $\delta_{n}$ value of $100 \mu \mathrm{m}$ is chosen for the reason that it is much larger than the characteristic diffusion lengths of both the Si solar cells.
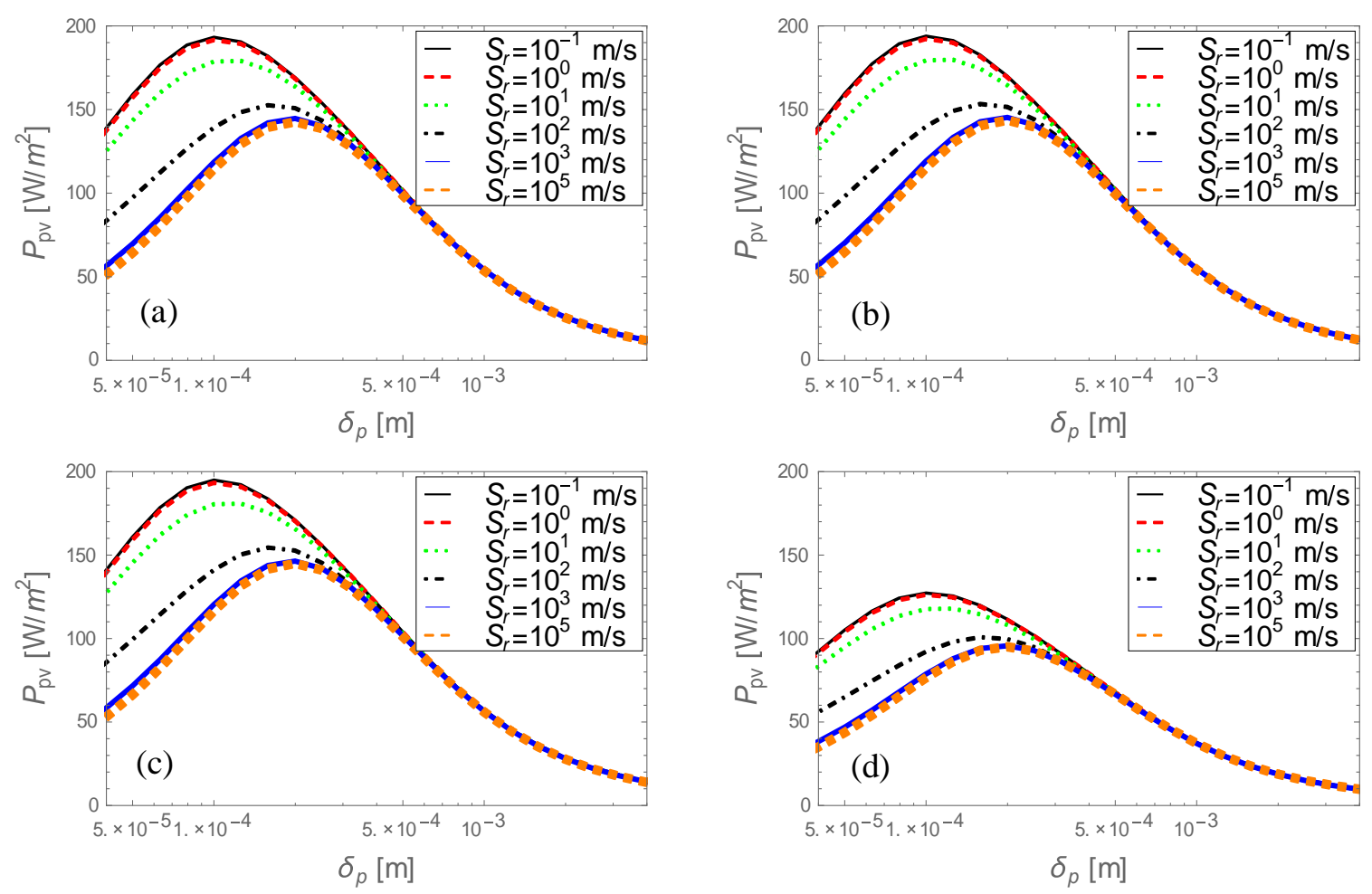

Fig. 2: Electric power $P_{p v}$ of the c-Si as a function of $\delta_{p}$ for a range of recombination velocities $S_{r}\left(10^{-1}-10^{5} \mathrm{~m} / \mathrm{s}\right)$, with $P_{s}=1000 \mathrm{~W} / \mathrm{m}^{2}$ and several $\delta_{n}$ : (a) $50 \mathrm{~nm}$, (b) $250 \mathrm{~nm}$, (c) $2 \mu \mathrm{m}$ and (d) $100 \mu \mathrm{m}$. 

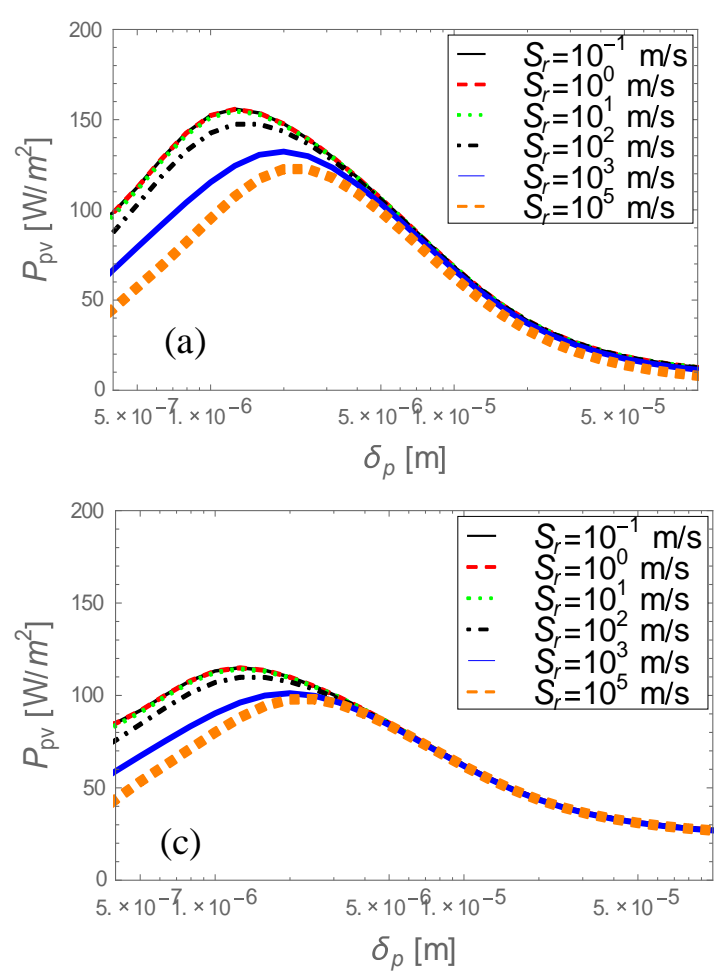
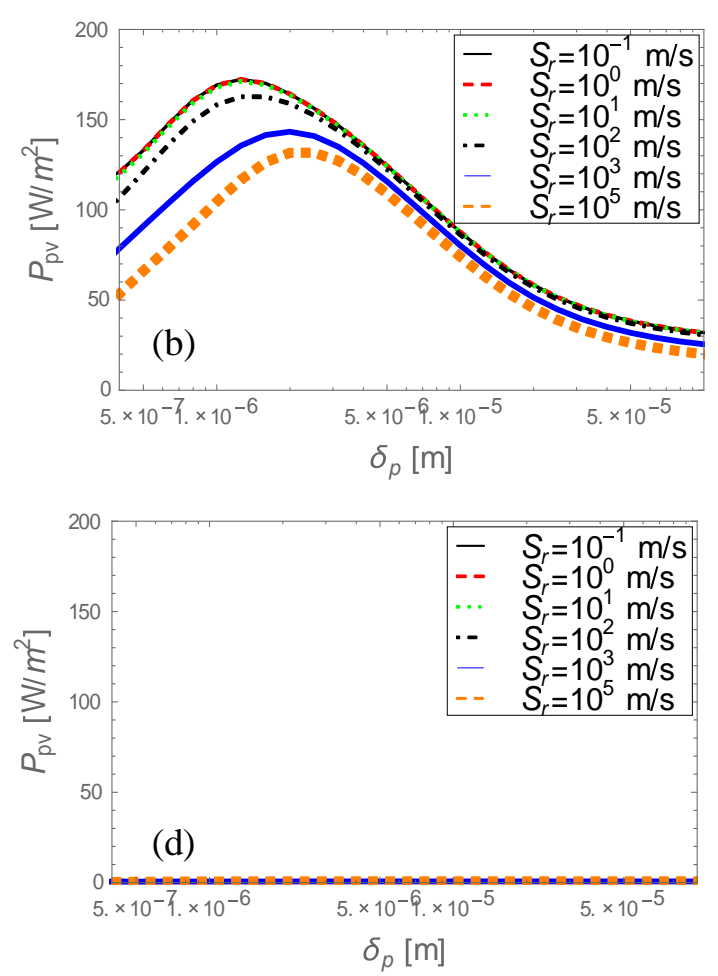

Fig. 3 Electric power $P_{p v}$ of the p-Si as a function of $\delta_{p}$ for a range of recombination velocities $S_{r}\left(10^{-1}-10^{5} \mathrm{~m} / \mathrm{s}\right)$, with $P_{s}=1000 \mathrm{~W} / \mathrm{m}^{2}$ and several $\delta_{n}$ : (a) $50 \mathrm{~nm}$, (b) $250 \mathrm{~nm}$, (c) $2 \mu \mathrm{m}$ and (d) $100 \mu \mathrm{m}$.

The optimal p-side thickness for the c-Si varies from 100 to $200 \mu \mathrm{m}$, for recombination velocities ranging from $10^{-1}$ to $10^{5} \mathrm{~m} / \mathrm{s}$, respectively, whatever the considered $\mathrm{n}$-side thicknesses. Noting that, for this given range of surface recombination velocities, this velocity is of more importance in a thinner device than in a thicker one, it is understandable that the photovoltaic efficiency is more affected by the surface recombination velocity at smaller thicknesses. In [57], it is said that the recombination velocity for an unprepared sample is of the order of $10^{2} \mathrm{~m} / \mathrm{s}$. After passivation of dangling bonds by an $\mathrm{HF} / \mathrm{H}_{2} \mathrm{O}$, it was lowered to about $\sim 10 \mathrm{~m} / \mathrm{s}$ [57]. We can see anyway that not much increase in the electric power output is observed in Figs. 2 and 3 when decreasing further the surface recombination velocity, so that we use in the following the typical value of $S_{r}=10 \mathrm{~m} / \mathrm{s}$. The corresponding optimal p-side thickness for $\mathrm{c}-\mathrm{Si}$ is $125 \mu \mathrm{m}$ and for $\mathrm{p}-\mathrm{Si}$ it is around $1 \mu \mathrm{m}$. These values also correspond to typical industrial values found in the literature [58,59], where it is confirmed that p-Si only needs a thickness of the order of $1 \mu \mathrm{m}$ and c-Si two orders more, due to the formers larger absorption coefficient [53]. The maximum electric power that defines these optimal thicknesses needs some reflection. Two opposing factors [60] govern the increase or decrease of the electric output: the shortcircuit current and the open-circuit voltage (see Eqs. (104)-(106)). Physically, these factors are influenced by the photonic energy absorption and the recombination rate, as is detailed in Section 4. Although a too thin cell will have much less bulk recombination (see Eqs. (89), (90), (98) and (99)), it will still not absorb enough photonic energy (see Eq. (58)), which will cause the electric output to decrease for thinner cells. At too thick cells, much larger than the characteristic diffusion length (see text under Eq. (93)), bulk recombination becomes predominant, even though a maximum of photonic energy is absorbed, and the electric output decreases as well. It is then understandable that a maximum can be found for intermediary thicknesses, as can be seen in Figs. 2 and 3. The difference in these intermediary thicknesses for $\mathrm{c}-\mathrm{Si}$ and $\mathrm{p}-\mathrm{Si}$ lies in the difference of the aforementioned characteristic diffusion lengths, determined by material properties. Figs. 2(d) and 3(d) shows that for large enough n-side 
thicknesses, the electric power (even at optimal p-side thickness) decreases. Fig. 4 shows the electric power as a function of the n-side thickness.
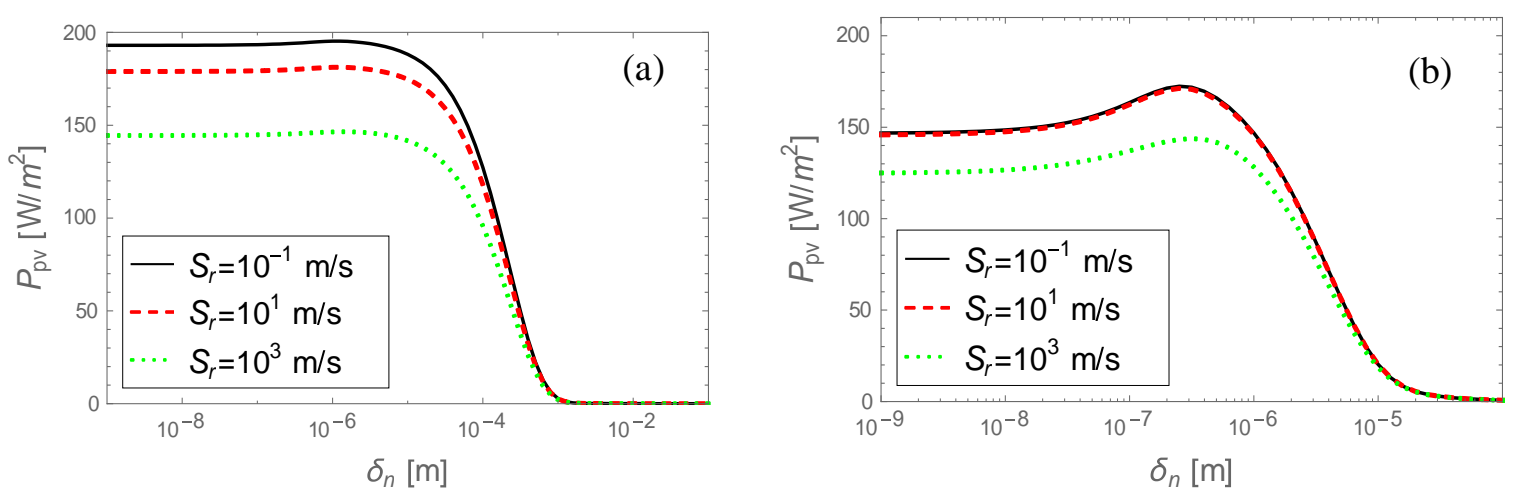

Fig. 4: Electric power $P_{p v}$ of the c-Si (a) and p-Si (b) as a function of the n-side thickness $\delta_{n}$ for a range of recombination velocities $S_{r}\left(10^{-1}, 10\right.$ and $\left.10^{3} \mathrm{~m} / \mathrm{s}\right)$ and corresponding optimal pside thicknesses $\delta_{p}(100,125$ and $200 \mu \mathrm{m})$, for $P_{s}=1000 \mathrm{~W} / \mathrm{m}^{2}$.

Fig. 4 shows indeed that for sufficiently large n-side thicknesses the electric power drops towards zero. The electric power starts already to drop considerably for n-side thicknesses comparable to the $\mathrm{p}$-side ones. The n-side being heavily doped, the recombination rate is much higher than in the p-side for the same thickness [48]. Therefore, the photo-generated free carriers recombine in the n-side, even before they can significantly be of any use in the p-side. This reduces considerably the photonic energy absorption in the p-side, which reduces the electric power. It is then understandable to reduce as much as possible the n-side thickness. For intermediate n-side thicknesses, however, a small maximum is visible in Fig. 4 . We have discussed the effect of too large n-side thicknesses. At too small n-side thicknesses, the recombination and photonic energy absorption will be insignificant with respect to those in the $\mathrm{p}$-side. At intermediate $\mathrm{n}$-side thicknesses, the photo-generation in the $\mathrm{n}$-side is significantly large enough to generate more electricity, but small enough to limit recombination and, therefore, also insignificant enough to interfere with the photo-generation in the p-side. In the foregoing, we proceed with the optimal n- and p-side thicknesses of $\delta_{n}=2 \mu \mathrm{m}$ and $\delta_{p}=125$ $\mu \mathrm{m}$ for c-Si and $\delta_{n}=250 \mathrm{~nm}$ and $\delta_{p}=1 \mu \mathrm{m}$ for $\mathrm{p}-\mathrm{Si}$, respectively.

\subsection{Influence of nanocomposite characteristics on thermoelectric efficiency}

Fig. 5 shows the thermoelectric efficiency as a function of the nanoparticle volume fraction for different nanoparticle radii and thermoelement lengths, where the heat comes from the c-Si photovoltaic cell. Fig. 6 shows the same results, but using the p-Si photovoltaic cell instead. 

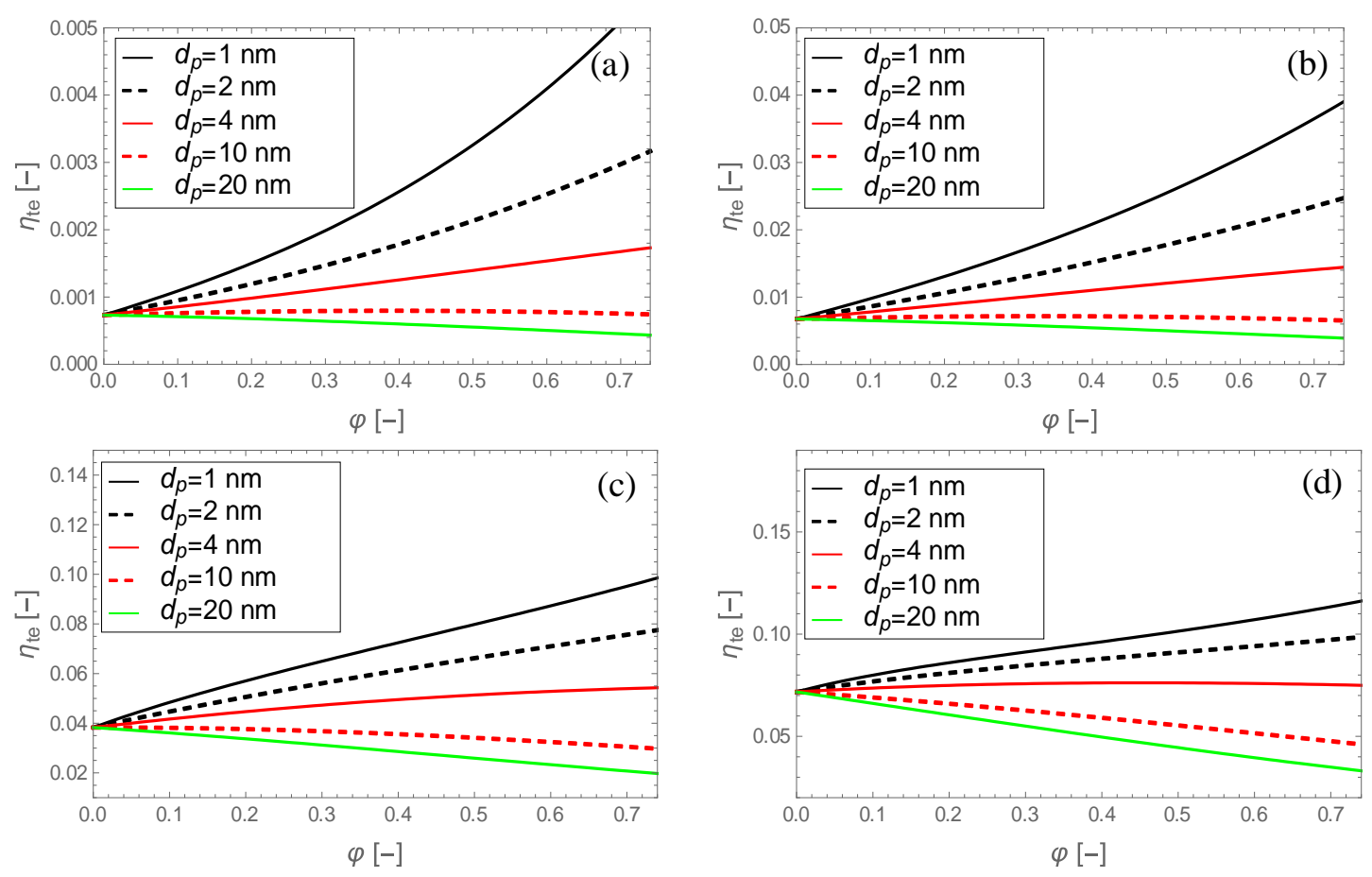

Fig. 5: Thermoelectric efficiency as a function of the nanoparticle volume fraction for different nanoparticle sizes $\left(d_{p}=1,2,4,10\right.$ and $\left.20 \mathrm{~nm}\right)$, a cooling velocity of $v_{c}=10 \mathrm{~m} / \mathrm{s}$ and thermoelectric element lengths of (a) $1 \mathrm{~mm}$, (b) $1 \mathrm{~cm}$, (c) $10 \mathrm{~cm}$ and (d) $1 \mathrm{~m}$. The heat comes from the photovoltaic c-Si cell.
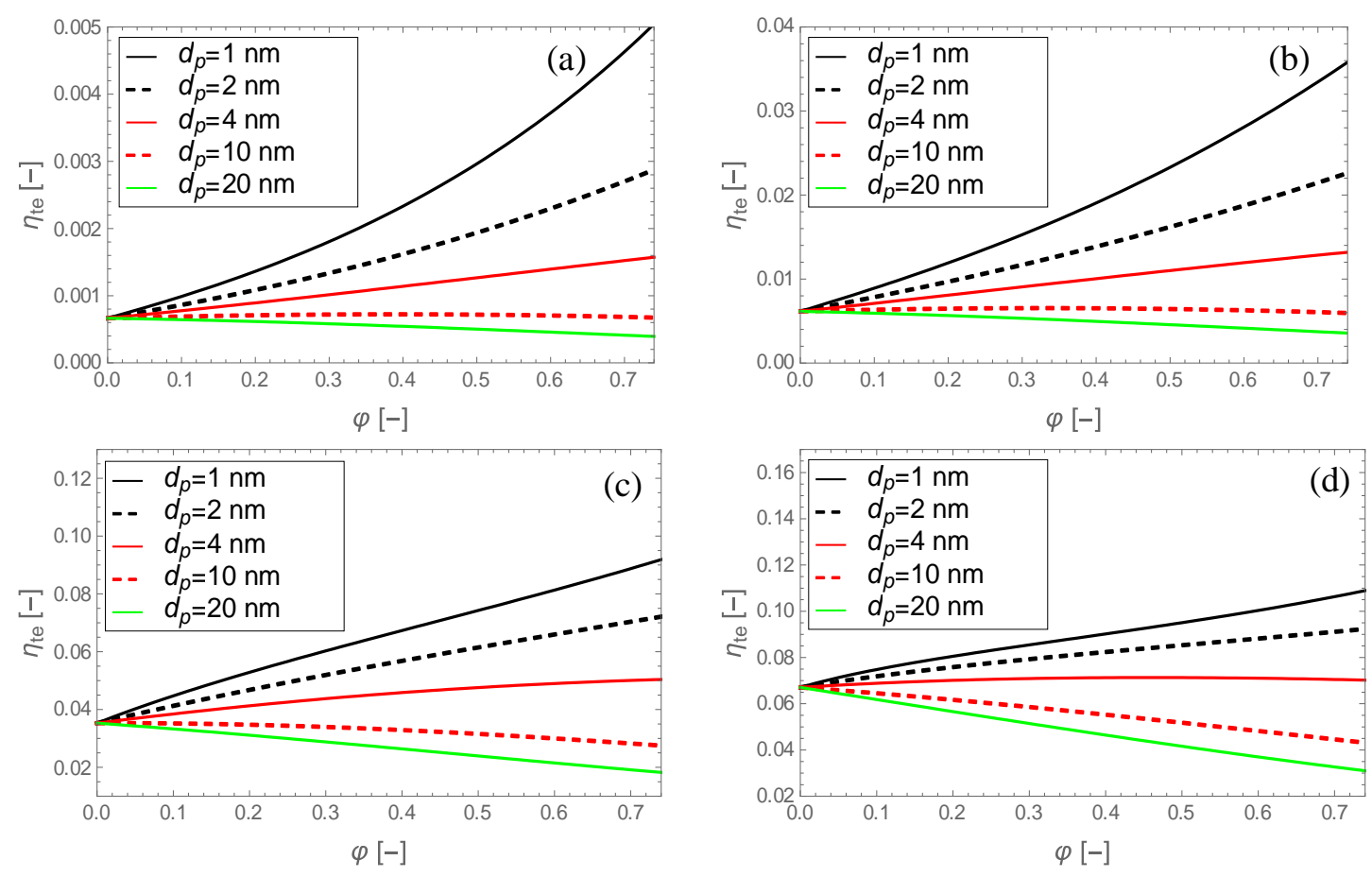

Fig. 6: Thermoelectric efficiency as a function of the nanoparticle volume fraction for different nanoparticle sizes $\left(d_{p}=1,2,4,10\right.$ and $\left.20 \mathrm{~nm}\right)$, a cooling velocity of $v_{c}=10 \mathrm{~m} / \mathrm{s}$ and thermoelectric element lengths of (a) $1 \mathrm{~mm}$, (b) $1 \mathrm{~cm}$, (c) $10 \mathrm{~cm}$ and (d) $1 \mathrm{~m}$. The heat comes from the photovoltaic p-Si cell. 
First of all, we can see a clear increase of the thermoelectric efficiency with increasing volume fraction, decreasing nanoparticle size and increasing thermoelectric element length. A decreasing thermoelectric nanoparticle size means that, especially if the size is of the order of magnitude of the mean free paths of phonons and electrons, the phonons and electrons are scattered more within the material [6]. As such the heat transport (due to phonon and electron transport as Eqs. (7a)-(7b) show) is slower and more electricity can be generated, increasing the figure of merit and thusly the thermoelectric efficiency. It is interesting to note that the figure of merit can be increased to almost 2.5 for the materials considered in this work. As an example, the figure of merit of the thermoelectric nanocomposite considered in this work is put against the volume fraction for various nanoparticle sizes is shown in Fig. 7.

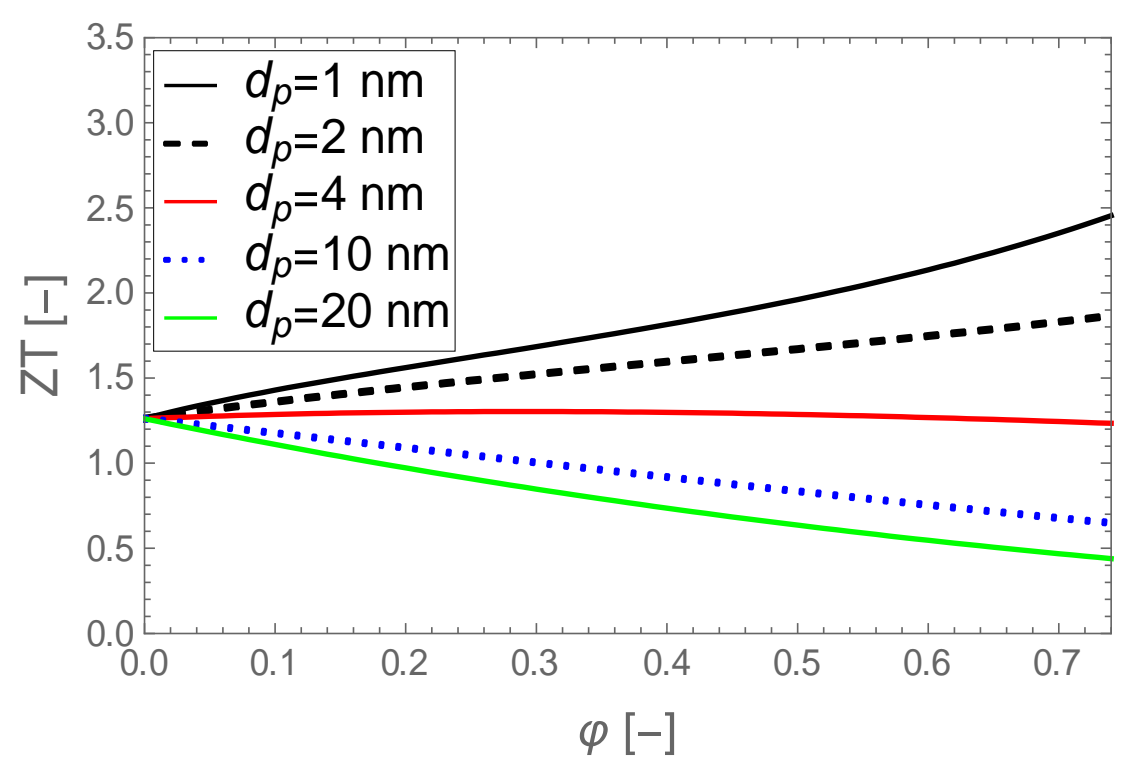

Fig. 7: Figure of merit of the nanocomposite of Section 6.1 as a function of the volume fraction for various nanoparticle sizes $\left(d_{p}=1,2,4,10\right.$ and $\left.20 \mathrm{~nm}\right)$.

Obviously, a higher volume fraction will only amplify the scattering effect at smaller nanoparticle sizes. As it can be seen, the highest efficiency is obtained for a volume fraction around 0.7. Note that due to geometric limitations, assuming that the nanoparticles are spheres, the highest theoretical volume fraction is $\varphi_{\max }=\pi / \sqrt{18}$. However, if the nanoparticle size is too large, the heat and electrical transport in the material is simply a function of the bulk values of the nanoparticle and matrix transports. The scattering effect will then be negligible (the Knudsen number tends to zero and Eq. (49) to the bulk value). As bulk values of the materials used for the nanoparticles in this work exhibit figures of merit lower than that of the matrix, a higher volume fraction will only decrease the thermoelectric efficiency. Fig. 7 shows indeed that for larger nanoparticle sizes, a higher volume fraction gives rise to a lower figure of merit. So, the effect of the volume fraction is not straightforward and depends on the nanoparticle size with respect to the mean free path, i.e. the Knudsen number. The length of the thermoelectric element influences the efficiency in that a longer element can absorb more heat and the temperature difference will be larger so that more electricity can be generated. Of course, from a theoretical point of view, an element's length going mathematically to zero will reduce the temperature difference across the element to zero as well. As the element's length increases, the efficiency will attain a maximum due to heat loss to the cooling device. This is shown in Fig. 8, where the thermoelectric efficiency is drawn against the thermoelectric element's length, with a volume fraction of 0.7 and a nanoparticle size of $2 \mathrm{~nm}$. For mathematical purposes, the range of thermoelectric lengths in Fig. 8 is extended beyond realistic limits. 

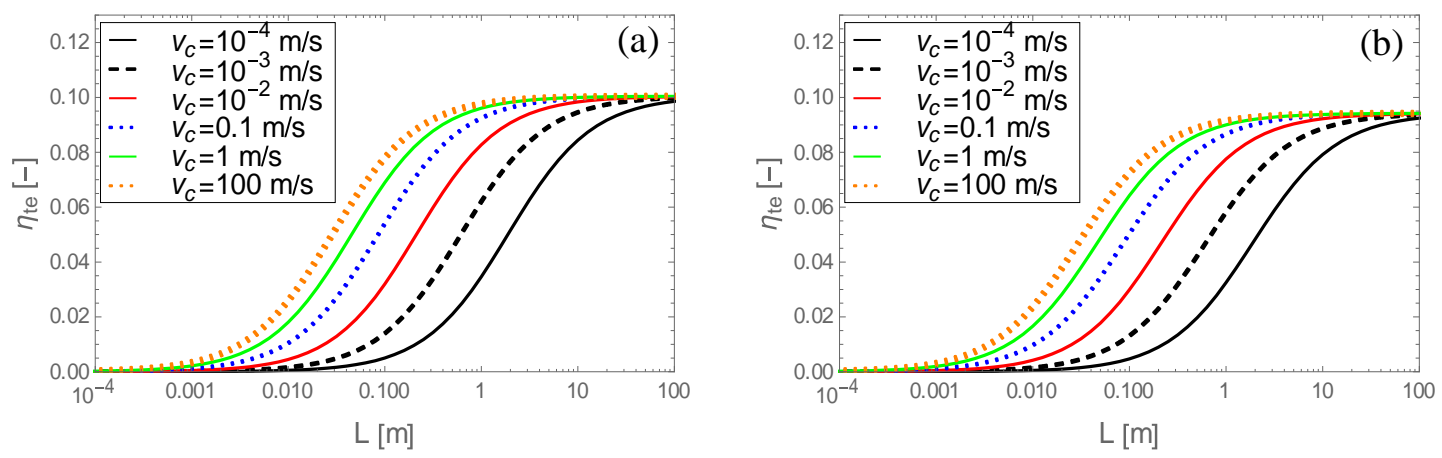

Fig. 8: Thermoelectric efficiency as a function of the thermoelement length for different cooling velocities, a volume fraction $\varphi=0.7$ and a nanoparticle size $d_{p}=2 \mathrm{~nm}$, where the heat comes from the photovoltaic c-Si cell (a) and p-Si cell (b).

To appreciate the effect of the cooling velocity on the thermoelectric efficiency, we present the results in Fig. 8 for various cooling velocities as well. It can be seen that the cooling velocities influence considerably the thermoelectric efficiency. A higher velocity will increase the heat transfer coefficient and the temperature difference across the thermoelectric element, increasing thereby the efficiency. The results in Fig. 8 are shown with nanoparticle size of $2 \mathrm{~nm}$. Smaller nanoparticle sizes will show even higher efficiencies. However, nanoparticles with sizes of the order of $1 \mathrm{~nm}$ and below begin to exhibit quantum confinement effects [61], not taken into account in the present model. Using a size of $1 \mathrm{~nm}$ is at the acceptable limit and deemed therefore still reasonable to present in this work. Nonetheless, to be at the safe side, we chose in Fig. 8 a nanoparticle size of $2 \mathrm{~nm}$ and will do so in the next subsection as well.

\subsection{Optimal hybrid opto-thermo-electric efficiency}

We have seen in the previous subsection that an optimal total photovoltaic device thickness is around $127 \mu \mathrm{m}$ and $1.25 \mu \mathrm{m}$ for c-Si and p-Si, respectively and that the highest, theoretically reasonable, thermoelectric efficiency is attained with a nanoparticle size of $2 \mathrm{~nm}$ and a volume fraction around 0.7. Choosing a thermoelectric element length of $10 \mathrm{~cm}$ (being practically reasonable [62] and close to maximum thermoelectric efficiency for higher cooling velocities), the results in the previous subsection suggest to increase the cooling velocity to a maximum. However, as the power for the cooling device is extracted directly from the electricity produced by the photovoltaic device, we can imagine that a too high cooling velocity will reduce the overall efficiency. It is therefore necessary to assess quantitatively the effect of the cooling velocity on the total efficiency. The results are shown in Fig. 9 for both the c-Si and p-Si photovoltaic devices connected to the thermoelectric element of the previous subsection.
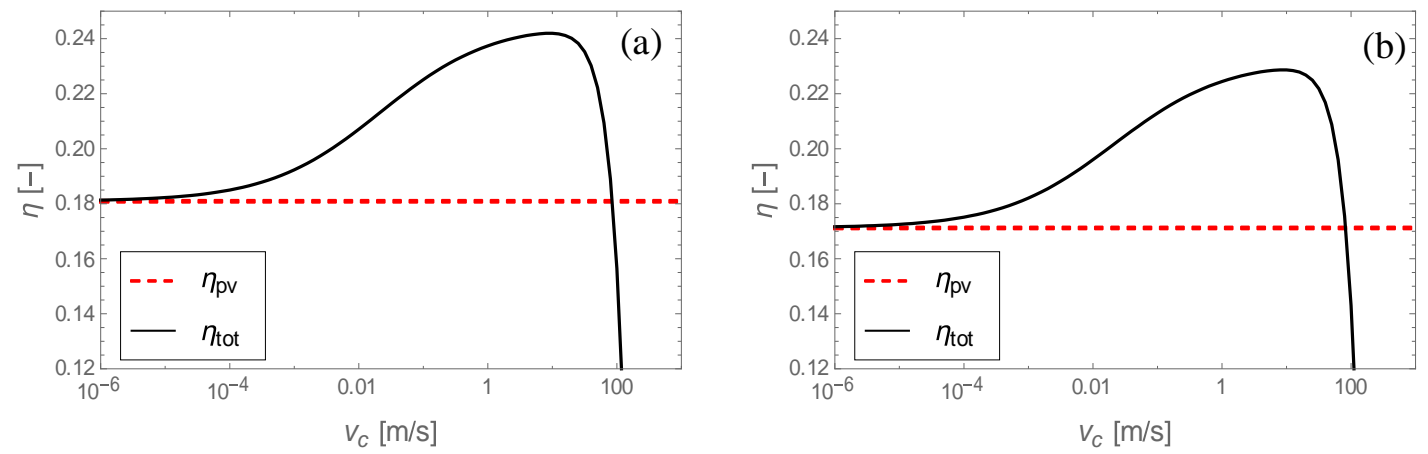
Fig. 9: Total hybrid efficiency as a function of the cooling velocity compared to the photovoltaic one. Two photovoltaic cells are compared: c-Si (a) and p-Si (b). The thermoelectric element in the hybrid systems is the one from Section 7.2 with $\varphi=0.7, d_{p}=2 \mathrm{~nm}$ and $L=0.1 \mathrm{~m}$.

Fig. 9 shows that indeed a too high cooling velocity makes the overall efficiency drop towards zero. An optimum cooling velocity appears to be around $10 \mathrm{~m} / \mathrm{s}$ for both hybrid systems, attaining overall efficiencies of almost $25 \%$.

\section{Conclusions}

This work presents a new analytical model describing the operation of a photovoltaicthermoelectric hybrid system assisted by a cooling device. The model is presented in three parts. The first part describes a thermoelectric model, where the material consists of nanoparticles embedded into a matrix. The thermoelectric element is composed out of an n-leg and a p-leg, made out of an n-type and p-type Bismuth Telluride matrix, respectively, with Antimony Telluride and Bismuth Selenide nanoparticles, respectively. The model in this work takes into account size-dependent thermoelectric properties, where non-local effects of heat transfer through phonons and electrons are important at nanoscales. These phenomena are extended to apply also for electric transfer as well as the Seebeck coefficient. The transport properties in the thermoelectric nanocomposite are derived in an original way from Extended Irreversible Thermodynamics. In the second part, a model is developed that describes the pn-junction in a photovoltaic cell, taking into account optical conversion to electricity, surface recombination effects and heat generation. Two photovoltaic materials are considered in this work: monocrystalline silicon (c-Si) and polycrystalline silicon (p-Si). The last part connects the two former ones with a cooling device (powered by electricity from the photovoltaic device) and deals with the heat management analysis of the hybrid system. It should be noted, however, that the photovoltaic model is also applicable to other materials that have typically a p-n junction (such as crystalline materials or inorganic materials of the III and IV groups). The thermoelectric model is also applicable to other types of nanocomposites.

An optimal total photovoltaic device size, obtained from an analysis on the effect of both the $\mathrm{n}$ - and p-side thicknesses on the electric power output, has been found being around $127 \mu \mathrm{m}$ and $1.25 \mu \mathrm{m}$ for $\mathrm{c}-\mathrm{Si}$ and $\mathrm{p}-\mathrm{Si}$, respectively, corresponding to typical values from the literature $[58,59]$. It has been shown that the thermoelectric efficiency is increased considerably for increasing volume fraction at the condition that the nanoparticle size is rather small, i.e. of the order smaller than $4 \mathrm{~nm}$, which corresponds to Knudsen numbers of the order of 1 or higher. For larger nanoparticle sizes, the opposite effect is obtained. The enhancement of the thermoelectric efficiency is explained on one side by an increase of the figure of merit, due to scattering effects that decrease the heat and electric transport properties, and on the other side by an increase of the temperature difference across the thermoelectric device. The latter is attained by a higher cooling velocity (a higher heat transfer coefficient between the thermoelectric element and the cooling device), the results of this work showing that the choice of the photovoltaic device hardly influences the thermoelectric efficiency. A study of the overall efficiency shows that a cooling velocity around $10 \mathrm{~m} / \mathrm{s}$ presents the highest overall efficiency, i.e. almost $25 \%$. Theoretically, if nanoparticle sizes and the surface recombination velocities could be lowered even further, overall efficiencies of almost $30 \%$ could be attained.

Finally, it can be said that it in this work it is shown that a rather comprehensive, but analytical model is able to describe electricity generation by both a photovoltaic device and a thermoelectric device. It is also useful for understanding the underlying mechanisms that can increase photovoltaic and/or photovoltaic-thermoelectric efficiencies, showing the interest of using nanotechnology for solar energy harvesting [63]. The present model has also shown how 
the photovoltaic efficiency can be enhanced by a cooled thermoelectric device, what the limitations are and how further enhancement could be achieved.

\section{Acknowledgements}

Financial support from BelSPo through the Prodex program is acknowledged.

\section{References}

[1] Su S, Wang Y, Wang J, Xu Z, Chen J. Material optimum choices and parametric design strategies of a photon-enhanced solar cell hybrid system. Sol. Energy Mat. Sol Cells 2014; 128: 112-118.

[2] Huang MJ, Eames PC, Norton B, Hewitt NJ. Natural convection in an internally finned phase change material heat sink for the thermal management of photovoltaics. Sol. Energy Mat. Sol Cells 2011; 95: 1598-1603.

[3] Liu W, Lucas K, McEnaney K, Lee S, Zhang Q, Opeil C, Chen G, Ren Z. Studies on the $\mathrm{Bi}_{2} \mathrm{Te}_{3}-\mathrm{Bi}_{2} \mathrm{Se}_{3}-\mathrm{Bi}_{2} \mathrm{~S}_{3}$ System for mid-temperature thermoelectric energy conversion. Energy Envir Sci 2013; 6: 552-560.

[4] Sumithra S, Takas NJ, Misra DK, Nolting WM, Poudeu PFP, Stokes KL. Enhancement in thermoelectric figure of merit in nanostructured $\mathrm{Bi}_{2} \mathrm{Te}_{3}$ with semimetal nanoinclusions. $\mathrm{Adv}$ Energy Mater 2011; 1: 1141-1147.

[5] Jou D, Sellitto A, Cimmelli VA. Multi-temperature mixture of phonons and electrons and nonlocal thermoelectric transport in thin layers. Int J Heat Mass Transf 2014; 71: 459-468.

[6] Machrafi H. An extended thermodynamic model for size-dependent thermoelectric properties at nanometric scales: application to nanofilms, nanocomposites and thin nanocomposite films. Appl Math Mod 2016; 40: 2143-2160.

[7] Zhang J, Xuan Y, Yang L. Performance of photovoltaic-thermoelectric hybrid systems. Energy 2014; 78: 895-903.

[8] Candadai AA, Kumar VP, Barshilia HC. Performance evaluation of a natural convectivecooled concentration solar thermoelectric generator coupled with a spectrally selective high temperature absorber coating. Sol Energy Mat Sol Cells 2016; 145: 333-341.

[9] Ju X, Wang ZF, Flamant G, Li P, Zhao WY. Numerical analysis and optimization of a spectrum splitting concentration photovoltaic-thermoelectric hybrid system. Sol Energy 2012; 86: 1941-1954.

[10] Yang DJ, Yin HM. Energy conversion efficiency of a novel hybrid solar system for photovoltaic, thermoelectric, and heat utilization. IEEE Trans Energy Convers 2011; 26: 662670.

[11] Benghanem M, Al-Mashraqi AA, Daffallah KO. Performance of solar cells using thermoelectric module in hot sites. Renewable Energy 2016; 89: 51-59.

[12] Li YL, Witharana S, Cao H, Lasfargues M, Huang Y, Ding YL. Wide spectrum solar energy harvesting through an integrated photovoltaic and thermoelectric system. Particuology 2014; 15: 39-44.

[13] Jou D, Casas-Vazquez J, Lebon G. Extended Irreversible Thermodynamics, 4rth edition, New York, Dordrecht, Heidelberg, London: Springer, 2010.

[14] Machrafi H. Heat transfer at nanometric scales described by extended irreversible thermodynamics. Comm Appl Ind Math 2016; 7: 177-195.

[15] Chávez-Urbiola EA, Vorobiev YV, Bulat LP. Solar hybrid system with thermoelectric generators. Sol Energy 2012; 86: 369-378.

[16] Tan M, Deng Y, Hao Y. Enhancement of thermoelectric properties induced by oriented nanolayer in $\mathrm{Bi}_{2} \mathrm{Te}_{2.7} \mathrm{Se}_{0.3}$ columnar films. Mat Chem Phys 2014; 146: 153-158. 
[17] Topic M, Campa A, Filipic M, Berginc M, Krasovec UO, Smole F, Optical and electrical modelling and characterization of dye-sensitized solar cells. Current Applied Physics 2010; 10:S425-S430.

[18] Omer SA, Infield DG. Design optimization of thermoelectric devices for solar power generation. Sol Energy Mater Sol Cells 1998; 53: 67-82.

[19] Hua YC, Cao BY. Ballistic-diffusive heat conduction in multiply-constrained nanostructures. Internat J Therm Sci 2016; 101: 126-132.

[20] Maxwell JC. Treatise on Electricity and Magnetism, second ed., Clarendon, Oxford, 1881.

[21] Bruggeman D. Berechnung verschiedener physikalischer Konstanten von heterogenen Substanzen. Anal Phys 1935; 24: 636-664.

[22] Nan CW, Birringer R, Clarke DR, Gleiter H. Effective thermal conductivity of particulate composites with interfacial thermal resistance. J Appl Phys 1997; 81: 6692-6699.

[23] Cattaneo C. Sulla conduzione del calore. Atti del Seminario Matematico e Fisico delle Università di Modena 1948; 3: 83-101.

[24] Dreyer W, Struchtrup H. Heat pulse experiments revisited. Continuum Mech Therm 2003; 3: 3-50.

[25] Hess S. On nonlocal constitutive relations, continued fraction rxpansion for the wave vector dependent diffusion coefficient. Z Naturforsch 1977; 32a: 678-684.

[26] Norton M, Gracia Amillo AM, Galleano R. Comparison of solar irradiance measurements using the average photon energy parameter. Solar Energy 2015; 120: 337-344.

[27] Khriachtchev L. Silicon Nanophotonics, basic principles, present status and perspectives, pan Stanford Publishing, Singapore (2009) p. 317.

[28] Palankovski V, Quay R. Analysis and simulation of heterostructure devices. SpringerVerlag, Vienna (2004).

[29] Richter A, Glunz SW, Werner F, Schmidt J, Cuevas A. Improved quantitative description of Auger recombination in crystalline silicon. Physical Review B 2012; 86: 165202.

[30] Cuevas A, Macdonald D. Measuring and interpreting the lifetime of silicon wafers. Sol. Energy 2003; 76: 255-262

[31] Alamo JA, Swanson RM. Modelling of minority-carrier transport in heavily doped silicon emitters. Solid-State Electronics 1987; 30: 1127-1136.

[32] Lu M. Silicon heterojunction solar cell and crystallization of amorphous silicon, $\mathrm{PhD}$ thesis University of Delaware 2008, p. 46.

[33] Salazar A, Apiñaniz E, Mendioroz A, Oleaga A. A thermal paradox: which gets warmer? Eur J Phys 2010; 31: 1053-1059.

[34] Balaj M, Roohi E, Akhlaghi H, Myong RS, Investigation of convective heat transfer through constant wall heat flux micro/nano channels using DSMC, Internat J Heat Mass Transfer 2014; 71: 633-638.

[35] Satterthwaete CB, Ure RW. Electrical and thermal properties of $\mathrm{Bi}_{2} \mathrm{Te}_{3}$. Phys Rev 1957; 108: 1164-1170.

[36] Lee WY, Park NW, Hong JE, Yoon SG, Koh JH, Lee SK. Effect of electronic contribution on temperature-dependent thermal transport of antimony telluride thin film. J. Alloys Compounds 2015; 620: 120-124.

[37] Lošt'ák P, Drašar C, Horák J, Zhou Z, Dyck JS, Uher C. Transport coefficients and defect structure of $\mathrm{Sb}_{2-\mathrm{x}} \mathrm{Ag}_{\mathrm{x}} \mathrm{Te}_{3}$ single crystals. J. Phys. Chem. Solids 2006; 67: 1457-1463.

[38] Cheng CH, Huang SY, Cheng TC. A three-dimensional theoretical model for predicting transient thermal behavior of thermoelectric coolers. Int J Heat Mass Trans 2010; 53: 20012011.

[39] Saci A, Battaglia JL, Kusiak A, Fallica R, Longo M. Thermal conductivity measurement of a $\mathrm{Sb}_{2} \mathrm{Te}_{3}$ phase charge nanowire. Appl Phys Lett 2014; 104: 263103. 
[40] Venkatasubramanian R. Lattice thermal conductivity reduction and phonon localizationlike behaviour in superlattice structures. Phys Rev B 2000; 61: 3091-3097.

[41] Herdt A. Exploring the electronic properties of novel spintronic materials by photoelectron spectroscopy. Forschungszentrum Jülich GmbH, Duisburg 2012, p. 77.

[42] Yang JY, Aizawa T, Yamamoto A, Ohta T. Thermoelectric properties of n-type $\left(\mathrm{Bi}_{2} \mathrm{Se}_{3}\right)_{\mathrm{x}}\left(\mathrm{Bi}_{2} \mathrm{Te}_{3}\right)_{1-\mathrm{x}}$ prepared by bulk mechanical alloying and hot pressing. $\mathrm{J}$ Alloys Compounds 2000; 312: 326-330.

[43] Bessas D, Töllner W, Aabdin Z, Peranio N, Sergueev I, Wille HC, Eibl O, Nielsch K, Hermann RP. Phonon spectroscopy in a $\mathrm{Bi}_{2} \mathrm{Te}_{3}$ nanowire array. Nanoscale 2013; 5: 1062910635.

[44] Sarma SD, Li Q. Many-body effects and possible superconductivity in the two-dimensional metallic surface states of three-dimensional topological insulators. Phys Rev B 2013; 88: 081404R.

[45] De Wette FW, Kulkarni AD. Phonon dispersion, phonon specific heat, and Debye temperature of high-temperature superconductors. Phys Rev B 1992; 46: 14922-14925.

[46] Lai YP, Chen HJ, Wu KH, Liu JM. Temperature-dependent carrier-phonon coupling in topological insulator $\mathrm{Bi}_{2} \mathrm{Se}_{3}$. Appl Phys Lett 2014; 105: 232110.

[47] Cho S, Butch NP, Paglione J, Fuhrer MS. Insulating behaviour in ultrathin bismuth selenide field effect transistors. Nanolett 2011; 11: 1925-1927.

[48] Wang F, Yu H, Li J, Wong S, Sun XW, Wang X, Zheng H. Design guideline of high efficiency crystalline Si thin film solar cell with nanohole array textured surface. J Appl Phys 2011; 109: 084306.

[49] Schmidt J, Kerr M, Altermatt PP. Coulomb-enhanced Auger recombination in crystalline silicon at intermediate and high-injection densities. J Appl Phys 2000; 88: 1494.

[50] Rein S, Glunz SW. Advanced lifetime spectroscopy. 13th Workshop on Crystalline Silicon Solar Cell Materials and Processes, NREL 2003, pp. 18-25.

[51] Da Y, Xuan Y. Role of surface recombination in affecting the efficiency of nanostructured thin-film solar cells. Optics Express 2013; 21: A1065-A1077.

[52] Jeffery LG. Handbook of photovoltaic science and engineering. Antonio Luque, 2004.

[53] Gutierrez EA, Deen MJ, Claeys CL. Low temperature electronics: physics, devices, circuits, and applications. Elsevier, 2001.

[54] Serra JM, Gamboa R, Vallera AM. Optical absorption coefficient of polycrystalline silicon with very high oxygen content. Mat Sci Eng B 1996; 36: 73-76.

[55] Hopkins PE, Reinke CM, Su MF, Olsson III RH, Shaner EA, Leseman ZC, Serrano JR, Phinney LM, El-Kady I. Reduction in the thermal conductivity of single crystalline silicon by phononic crystal patterning. Nano Lett 2011; 11: 107-112.

[56] Uma S, McConnell AD, Asheghi M, Kurabayashi K, Goodson KE. Temperaturedependent thermal conductivity of undoped polycrystalline silicon layers. Int $\mathbf{J}$ Thermophys 2001; 22: 605-616.

[57] Baek D, Rouvimov S, Kim B, Jo TC, Schroder DK. Surface recombination velocity of silicon wafers by photoluminescence. Appl Phys Lett 2005; 86: 112110.

[58] Mishima T, Taguchi M, Sakata H, Maruyama E. Development status of high-efficiency HIT solar cells. Solar Energy Mat Solar Cells 2011; 95: 18-21.

[59] Yamamoto K, Yoshimi M, Suzuki T, Okamoto Y, Tawada Y, Nakajima A. Thin film polySi solar cell with "Star Structure" on glass substrate fabricated at low temperature. Conf. Record 26th. IEEE Photovoltaic Specialists Conf., Anaheim, IEEE Press, Piscataway, 575-580.

[60] Sah RLY, Yamakawa KA, Lutwack R. Effect of thickness on silicon solar cell efficiency. Electron Devices, IEEE Trans. 1982; 29: 903-908.

[61] Lebon G, Machrafi H. Thermal conductivity of tubular nanowire composites based on a thermodynamical model. Physica E 2015; 71: 117-122. 
[62] Hashim H, Bomphrey JJ, Min G. Model for geometry optimization of thermoelectric devices in a hybrid PV/TE system. Renew Energy 2016; 87: 458-463.

[63] Abdin Z, Alim MA, Saidur R, Islam MR, Rashmi W, Mekhilef S, Wadi A. Solar energy harvesting with the application of nanotechnology. Renew Sustain Energy Rev 2013; 26: 837852 . 